Article

\title{
Discrete-Time Attitude Tracking Synchronization for Swarms of Spacecraft Exploiting Interference
}

\author{
Peiran Li ${ }^{1, *} \mathbb{C}$, Xin Wen ${ }^{1}$, Mohong Zheng ${ }^{1}$, Haiying Liu ${ }^{1,2}$, Dizhi Long ${ }^{1}$ and Yuping Lu ${ }^{1}$ \\ 1 Department of Astronautics, Nanjing University of Aeronautics and Astronautics, Nanjing 211100, China; \\ xinw@nuaa.edu.cn (X.W.); mohongzheng@nuaa.edu.cn (M.Z.); liuhaiying@nuaa.edu.cn (H.L.); \\ longdizhi@nuaa.edu.cn (D.L.); yplac@nuaa.edu.cn (Y.L.) \\ 2 Nanjing Center for Applied Mathematics, Nanjing 211135, China \\ * Correspondence: peiranli@nuaa.edu.cn
}

check for updates

Citation: Li, P.; Wen, X.; Zheng, M.; Liu, H.; Long, D.; Lu, Y. Discrete-Time Attitude Tracking Synchronization for Swarms of Spacecraft Exploiting Interference. Aerospace 2022, 9, 134. https:// doi.org/10.3390/aerospace 9030134

Academic Editor: Angelo Cervone

Received: 16 December 2021

Accepted: 1 March 2022

Published: 3 March 2022

Publisher's Note: MDPI stays neutral with regard to jurisdictional claims in published maps and institutional affiliations.

Copyright: (C) 2022 by the authors. Licensee MDPI, Basel, Switzerland. This article is an open access article distributed under the terms and conditions of the Creative Commons Attribution (CC BY) license (https:// creativecommons.org/licenses/by/ $4.0 /)$.

\begin{abstract}
The attitude tracking synchronization control of an orbit-predetermined leader-follower spacecraft swarm for the space moving target is discussed in this paper. The information exchange between all spacecraft is assumed to be discrete in time and on the undirected connected graph. Moreover, due to the demand for saving communication resources, wireless interference has been utilized, which allows all the neighbors of a spacecraft to access the same channel frequency spectrum simultaneously. Then the backstepping control algorithm is designed to let the spacecraft $(\beta, A)$ practically stably synchronize their states and track a time-varying trajectory in the presence of unknown fading channels. Finally, simulation is provided to verify that using the proposed control scheme, the attitude tracking synchronization can be achieved with high precision.
\end{abstract}

Keywords: aerospace; discrete event systems; nonlinear systems; synchronization; control over communications

\section{Introduction}

In recent years, small spacecraft, even micro and nano spacecraft cooperating to complete complex space missions, have attracted a lot of attention [1,2]. For example, three CubeSats were used to assemble a space telescope in the Autonomous Assembly of a Reconfigurable Space Telescope (AAReST) mission, and a swarm (hundreds to thousands) of 100 g-class femto-spacecraft were planned to launch for distributed sensor networks in The Swarms of Silicon Wafer Integrated Femtosatellites (SWIFT) mission [3]. Compared with using the traditional large spacecraft to complete complex missions, the spacecraft swarm consisting of multiple simple small spacecraft is more flexible, reliable, and robust. However, one of the main challenges that constrain spacecraft swarm missions is the design of the communication scheme when a large number of spacecraft are involved in the swarm or the inter-spacecraft communication channel is limited [4]. Due to the constraint of satellite quality, the capability of the onboard computer and communication system is really limited. Physically, spacecraft use low bandwidth to communicate within the swarm and high bandwidth for data transfer back to Earth. Low bandwidth of communications will limit the availability and timeliness of information transfer among the spacecraft. As a result, if the onboard computer requires more information than the spacecraft can transmit over the communications channel, the spacecraft would not survive in the complex space environment [5]. Moreover, it has been well recognized that discrete-time communication may cause undesirable dynamic network behaviors such as instability [6-9]; therefore, it is another challenge to design the control scheme for the discrete-time spacecraft swarm.

Traditionally, two kinds of methods can be used to save communication resources, which are decentralizing communication topology $[10,11]$ and reducing interactive information $[12,13]$. In decentralizing communication topology, every spacecraft is designed to make decisions based on the local information obtained from its neighbors according 
to geographical distance, sensor distribution, or security problems. There are numerous studies on how to design the local interaction rules for swarms so that they can exhibit a desired collective behavior. The approach to design the communication links to restore convexity for strongly connected systems based on the graph theory has been illustrated in [10], and a design method of time-varying information links has been proposed for more general systems. The instrumental role of the algebraic connectivity of graphs and digraphs in the analysis of consensus algorithms has been demonstrated in [11]. Based on these results, the consensus problem for general linear multi-agent systems on a general graph has been studied in [14-16]. The interacting heterogeneous linear multi-agent systems synchronization has been achieved in [17]. Consensus algorithms for nonlinear Euler-Lagrange systems have been researched in [18,19]. Furthermore, coordinated attitude control for spacecraft tracking a constantly desired attitude has been researched in [20,21], and coordinated attitude control for spacecraft tracking the common time-varying reference states modeled by sine and cosine functions has been discussed in [22,23]. In reducing interactive information, the amount of information required will be reduced by the optimization of control scheme. Mei J et al. [12] researched the formation control algorithm without speed information. Whilst Zhao S et al. [13] presented bearing-based formation stabilization of which only direction information is required.

The above-mentioned two strategies are based on the fact that the information transmitting is performed using the traditional orthogonal channel access method (OCAM), in which the information exchange is agent-to-agent and avoids interference by time or frequency multiplexing [4]. Nevertheless, when the number of spacecraft in the swarm is huge, with these methods, the pressure of the wireless channel is rising at least proportionally to the number of spacecraft. In 2018, harnessing the interference of the wireless channel for consensus problems has been pioneered by Molinari et al. [24,25]. By utilizing interference, multiple agents are allowed to access the same channel frequency spectrum simultaneously, which is faster than time-multiplexing OCAM, and more efficient than frequency-multiplexing OCAM. Despite its great potential for improving the communication efficiency of cooperative control systems, there is no literature attempting to apply information interference in synchronization control of complex nonlinear systems. Hence, the design of the communication scheme exploiting interference constitutes the first main focus of this paper.

Besides, in practical applications, as the spacecraft in the swarm need to acquire information from the neighbors and then update their states, communication between spacecraft should be modeled as discrete-time networks. The interval between two adjacent update instants is a challenge for meeting the requirements of convex calculation. Thus, a rather challenging problem in spacecraft attitude tracking synchronization control is discrete synchronization, which has recently begun to receive initial research attention. For example, the discrete synchronization problem of single-integrator networked multi-agent system, non-holonomic agents system, and lower triangular heterogeneous multi-agent system has been solved in [6-8], respectively; furthermore, the range of the sampling period has been obtained. Moreover, a discrete leader-follower synchronization problem has been addressed in [9], where the leader is governed by general double-integrator dynamics and the followers are Euler-Lagrange systems. However, as reference [26] illustrated, most of the existing discrete theoretical results are focused on the single-integrator, doubleintegrator, nonlinear oscillators, nonholonomic mobile robots, typically complex networks, and the typical Euler-Lagrange dynamic system. The design of the discrete control scheme for the spacecraft swarm attitude tracking synchronization system is still worthy of some attention, which constitutes the second main focus of this paper.

The main contribution of this paper is to present a control law for spacecraft attitude tracking synchronization under the assumption of discrete-time communication. More precisely, a discrete-time control algorithm is proposed to drive a group of spacecraft to synchronize their attitudes and track a moving target with attitude-orbit coupling. In addition, in this paper, information interference is used for information transmission solving 
the challenge of limited communication in spacecraft swarm. Compared with the frequencymultiplexing OCAM, the communication scheme can save the channel resource as multiple agents can access the same channel frequency spectrum. As a result, different from the existing literatures that deal with limited communication by decentralizing communication topology and reducing interactive information, the communication scheme in this paper can save communication resources in proportion to the number of spacecraft. The control law in this paper also deals with the unknown channel attenuation problem when exploiting information interference.

This paper is organized as follows: at first, in Section 2, the problem description in natural language and preliminary knowledge about spacecraft attitude kinematics and dynamics, graph, information interference and stabilization theory of sampled-data nonlinear systems is summarized and the problem description in mathematical language is given. Then, in Section 3, we derive the control algorithm and prove its effectiveness. Moreover, numerical simulation is shown in Section 4. Finally, the conclusion is stated in Section 5.

Notations: Throughout this paper, $\boldsymbol{R}$ denotes the set of real numbers. $\boldsymbol{R}_{>0}$ denotes the set of positive real numbers. $N_{>0}$ denotes the set of positive integers. The set of nonnegative real numbers is $\boldsymbol{R}_{>0}$ and the set of nonnegative integers is $\boldsymbol{N}_{>0}$. Given a matrix $\boldsymbol{A} \in \boldsymbol{R}^{n \times m}$, the entry in position $(i, j)$ is $[\boldsymbol{A}]_{i, j} . \mathbf{I}_{n}$ stands for the n-dimensional identity matrix. $\mathbf{0}_{n \times m}$ denotes the matrix of zeros with $n$ rows and $m$ columns. $\otimes$ represents the Kronecker product. For matrix $\boldsymbol{A} \in \boldsymbol{R}^{n \times n}$ and $\boldsymbol{B} \in \boldsymbol{R}^{n \times n}, \boldsymbol{A} \geq \boldsymbol{B}$ means that $\boldsymbol{A}-\boldsymbol{B}$ is positive semidefinite, and $\boldsymbol{A}>\boldsymbol{B}$ means that $\boldsymbol{A}-\boldsymbol{B}$ is positive definite. Given constant sampling intervals $\left[t_{k}, t_{k+1}\right), \forall t_{k} \in \boldsymbol{R}_{\geq 0}, k \in \boldsymbol{N}_{\geq 0}$, with $\Delta t=t_{k+1}-t_{k}$, if a function $f(\cdot)$ is updated at sampling instants, i.e., during the period $\left[t_{k}, t_{k+1}\right), f(t) f\left(t_{k}\right), f\left(t_{k}\right)$ is abbreviated as $f(k)$. A continuous function $\alpha(\cdot):[0, \mathrm{a}) \rightarrow \boldsymbol{R}_{\geq 0}$ is said to belong to class $\mathcal{K}$ if $\alpha(\cdot)$ is strictly increasing and subject to $\alpha(0)=0$. A continuous function $\beta(\cdot):[0, \mathrm{~b}) \rightarrow \boldsymbol{R}_{\geq 0}$ is said to belong to class $\mathcal{K}_{\infty}$ if it belongs to class $\mathcal{K}, \mathrm{b}=\infty$ and $\lim _{r \rightarrow \infty} \beta(\infty)=\infty$. A function $\gamma: \boldsymbol{R}_{\geq 0} \times \boldsymbol{R}_{\geq 0} \rightarrow \boldsymbol{R}_{\geq 0}$ is of class $\mathcal{K} \mathfrak{w}$ if $\gamma(\cdot, t)$ is of class $\mathcal{K} \forall t \in \boldsymbol{R}_{\geq 0}$ and $\gamma(s, \cdot)$ is decreasing to zero for each $\mathrm{s} \in \boldsymbol{R}_{>0}$.

\section{Problem Description and Preliminary Knowledge}

The space observation applications need high accurate alignment between spacecraft and the moving target. In engineering, each swarm mission can have hundreds or even thousands of cooperating spacecraft working in teams. Some spacecraft will be responsible for target positioning, others will major in data collection. In this paper, we consider attitude tracking synchronization for swarms of spacecraft without relative position control. The attitudes of spacecraft need to track moving targets synchronously for observation. To model the spacecraft performing target positioning, a virtual leader spacecraft (LS) is considered in this paper. The attitude of the LS is calculated by the orbit elements of itself and the orbit elements of the target, i.e., the goal of the LS is to track the moving target and maneuver to point to the target, and then the whole swarm needs to achieve attitude synchronization to observe. Figure 1 depicts this scenario. It is worth mentioning that the attitude tracking synchronization problem in this paper is the simplification of the spacecraft attitude cooperative tracking problem, details refer to Section 2.5.

In the following, the mathematical description of spacecraft kinematics and dynamics, graph and the preliminary knowledge of wireless interference and stabilization theory of sampled-data nonlinear systems are presented at first, then the mathematic model of the problem description is stated clearly. 


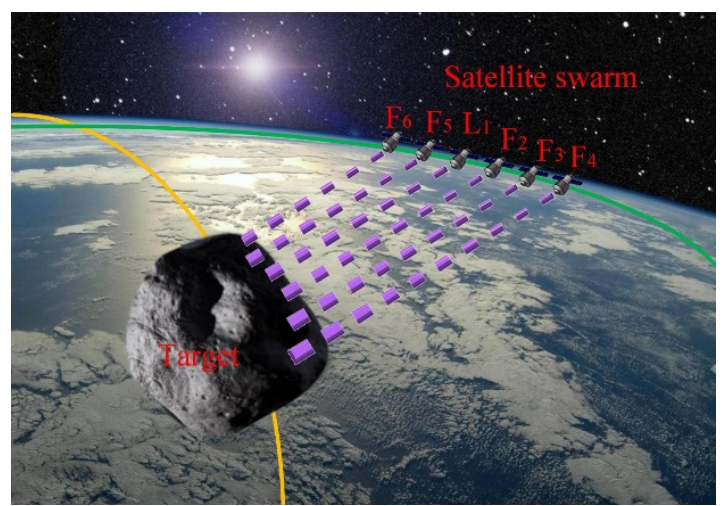

Figure 1. Scenario for the tracking and observing swarm.

\subsection{Kinematics and Dynamics}

\subsubsection{Axis Frame}

As shown in Figure 2, there are three commonly used axis frames for determining the attitude of a spacecraft:

- Earth Centered Inertial Frame, $O_{\mathrm{I}} X_{\mathrm{I}} Y_{\mathrm{I}} Z_{\mathrm{I}}$. The origin of $O_{\mathrm{I}} X_{\mathrm{I}} Y_{\mathrm{I}} Z_{\mathrm{I}}$ is fixed to the barycenter of the Earth. The $X_{\mathrm{I}} \mathrm{O}_{\mathrm{I}} Y_{\mathrm{I}}$ plane coincides with the equatorial plane. The $\mathrm{O}_{\mathrm{I}} Z_{\mathrm{I}}$ axis points to the North Pole, the $\mathrm{O}_{\mathrm{I}} X_{\mathrm{I}}$ axis points to the vernal equinox and the $O_{\mathrm{I}} Y_{\mathrm{I}}$ axis is determined according to the right-hand rule.

- Spacecraft Body Fixed Frame, $O_{\mathrm{B}} X_{\mathrm{B}} Y_{\mathrm{B}} Z_{\mathrm{B}}$. It is fixed on the spacecraft, and the frame origin corresponds to the center of mass. The $O_{B} Z_{B}$ axis points along the longitudinal axis of the spacecraft. The $O_{\mathrm{B}} X_{\mathrm{B}}$ axis and the $O_{\mathrm{B}} Y_{\mathrm{B}}$ axis lie, respectively, along the other two principal axes of the spacecraft according to the right-hand rule.

- Desired Imaging Frame, $O_{D} X_{D} Y_{D} Z_{D}$. The origin of $O_{D} X_{D} Y_{D} Z_{D}$ corresponds to the center of mass of the spacecraft. The $O_{\mathrm{D}} Z_{\mathrm{D}}$ axis points to the target. The $O_{\mathrm{D}} X_{\mathrm{D}}$ axis is determined by $Z_{D} \times\left(-Y_{B}\right)$, where $Z_{D}$ and $Y_{B}$ are the unit vectors of the $O_{D} Z_{D}$ axis and the $O_{\mathrm{B}} Y_{\mathrm{B}}$ axis. Then the $O_{\mathrm{D}} Y_{\mathrm{D}}$ axis is determined according to the right-hand rule.

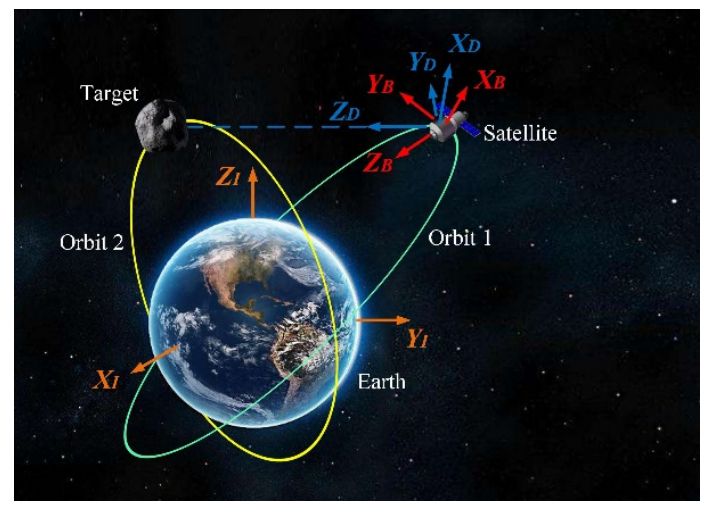

Figure 2. Three-axis frames for spacecraft attitude determination.

\subsubsection{Quaternion Kinematics}

Throughout this paper, the attitude of the spacecraft labeled $i \in\{1 \ldots n\}$ is described (in $O_{\mathrm{I}} X_{\mathrm{I}} Y_{\mathrm{I}} Z_{\mathrm{I}}$ ) by quaternion $\boldsymbol{Q}_{i}=\left[q_{0, i}, \boldsymbol{q}_{i}^{\mathrm{T}}\right]^{\mathrm{T}}$, where $q_{0, i} \in \boldsymbol{R}$ and $\boldsymbol{q}_{i}=\left[q_{1, i}, q_{2, i}, q_{3, i}\right]^{\mathrm{T}} \in \boldsymbol{R}^{3}$ denote, respectively, the scalar part and the vector part of this quaternion. According to Euler's rotation theorem, in three-dimensional space, any displacement of a rigid body that keeps at least one of its points fixed can be described by a rotation at angle $\alpha$ around a fixed axis $\left[e_{x}, e_{y}, e_{z}\right]$, and the axis $\left[e_{x}, e_{y}, e_{z}\right]$ should pass through these fixed points. One can find a full characterization of the quaternion as a function of $\alpha$ and $\left[e_{x}, e_{y}, e_{z}\right]$ in [27]. In particular, the scalar part of a quaternion is $q_{0, i}:=\cos \left(\frac{\alpha}{2}\right)$ whilst the vector part of a 
quaternion is $\boldsymbol{q}_{i}:=\left[e_{x} \sin \left(\frac{\alpha}{2}\right), e_{y} \sin \left(\frac{\alpha}{2}\right), e_{z} \sin \left(\frac{\alpha}{2}\right)\right]^{\mathrm{T}}$. Given a quaternion $\boldsymbol{Q}_{i}=\left[q_{0, i}, \boldsymbol{q}_{i}^{\mathrm{T}}\right]^{\mathrm{T}}$, its conjugate quaternion is denoted by $\bar{Q}_{i}=\left[q_{0, i},-\boldsymbol{q}_{i}^{\mathrm{T}}\right]^{\mathrm{T}}$. For any initial quaternion $\boldsymbol{Q}_{i}$ and maneuver quaternion $Q_{m, i}=\left[q_{0, m i}, q_{1, m i}, q_{2, m i}, q_{3, m i}\right]^{\mathrm{T}}$, and the final quaternion $=Q_{k, i}=$ $\left[q_{0, k i}, q_{1, k i}, q_{2, k i}, q_{3, k i}\right]^{\mathrm{T}}$ can be expressed as [28].

$$
\begin{aligned}
\boldsymbol{Q}_{k, i} & =\left[\begin{array}{cccc}
q_{0, m i} & -q_{1, m i} & -q_{2, m i} & -q_{3, m i} \\
q_{1, m i} & q_{0, m i} & -q_{3, m i} & q_{2, m i} \\
q_{2, m i} & q_{3, m i} & q_{0, m i} & -q_{1, m i} \\
q_{3, m i} & -q_{2, m i} & q_{1, m i} & q_{0, m i}
\end{array}\right]\left[\begin{array}{l}
q_{0, i} \\
q_{1, i} \\
q_{2, i} \\
q_{3, i}
\end{array}\right] \\
& =[\overbrace{\boldsymbol{Q}_{m, i}^{\times}}^{\times}] \boldsymbol{Q}_{i} .
\end{aligned}
$$

Remark 1. Quaternions $[1,0,0,0]^{T}$ and $[-1,0,0,0]^{T}$ correspond to the same Euler angle $[0,0,0]^{T}$.

The attitude angular velocity of spacecraft labeled $i \in 1 \ldots n$ is represented by $\boldsymbol{\omega}_{i}=\left[\omega_{1, i}, \omega_{2, i}, \omega_{3, i}\right]^{\mathrm{T}} \in \boldsymbol{R}^{3}$. According to [29], the equation describing the kinematics of spacecraft $i$ is

$$
\dot{Q}_{i}=\frac{1}{2} \Xi\left[Q_{i}\right] \omega_{i}
$$

where

$$
\Xi\left[\boldsymbol{Q}_{i}\right]:=\left[\begin{array}{ccc}
-q_{1, i} & -q_{2, i} & -q_{3, i} \\
q_{0, i} & -q_{3, i} & q_{2, i} \\
q_{3, i} & q_{0, i} & -q_{1, i} \\
-q_{2, i} & q_{1, i} & q_{0, i}
\end{array}\right]
$$

\subsubsection{Attitude Dynamics}

As [30] introduced, dynamics of spacecraft $i \in\{1 \ldots n\}$ is

$$
J_{i} \dot{\omega}_{i}+\omega_{i} \times J_{i} \omega_{i}=T_{i}
$$

where $\boldsymbol{J}_{i} \in \boldsymbol{R}^{3 \times 3}$ represents the momentum of inertia of spacecraft $i$ and $\boldsymbol{T}_{i} \in \boldsymbol{R}^{3}$ denotes the control torque for spacecraft $i$.

\subsubsection{Orbit Elements}

The orbit elements of a celestial body are denoted by Semi-major axis $a_{\mathcal{c}}$, Eccentricity $e_{c}$, Inclination $i_{c}$, Right Ascension of the Ascending Node $\Omega_{c}$, Argument of Perigee $\omega_{c}$ and Initial true anomaly $\Theta_{c 0}$, respectively. Then the position vector $\boldsymbol{P}_{c}=\left[P_{\mathrm{x}, c}, P_{\mathrm{y}, c}, P_{\mathrm{z}, c}\right]^{\mathrm{T}} \in \boldsymbol{R}^{3}$ of the celestial body in $O_{\mathrm{I}} X_{\mathrm{I}} Y_{\mathrm{I}} Z_{\mathrm{I}}$ is obtained by the following process

1. Calculate the orbital period $T_{c}: T_{\mathcal{C}}=2 \pi \sqrt{\frac{a_{c}^{3}}{398,600}}(s)$,

2. Calculate the mean anomaly $\mathrm{M}_{c}: \mathrm{M}_{c}=\frac{2 \pi}{T_{c}} t$,

3. Calculate the eccentric anomaly $E_{c}$ by solving the Kepler equation: $\mathrm{M}_{\mathcal{c}}=E_{c}-$ $e_{c} \sin \left(E_{c}\right)$,

4. Calculate the true anomaly $\Theta_{c}: \Theta_{c}=2 \tan ^{-1}\left(\tan \frac{E_{c}}{2} \sqrt{\frac{1+e_{c}}{1-e_{c}}}\right)+\Theta_{c 0}$,

5. Calculate the distance between the barycenter of the target and the barycenter of the Earth $r_{c}: r_{c}=a_{c} \frac{1-e_{c}^{2}}{1+e_{c} \cos \left(\Theta_{c}\right)}$,

6. Calculate $\boldsymbol{P}_{c}: \boldsymbol{P}_{c}=\boldsymbol{C}_{\mathrm{BI}} r_{c} \boldsymbol{z}$, where 


$$
\begin{gathered}
C_{\mathrm{BI}}=\left(C_{\Theta_{c}} C_{\omega_{c}} C_{i_{c}} C_{\Omega_{c}}\right)^{\mathrm{T}} \\
C_{\Theta_{c}}=\left[\begin{array}{ccc}
\cos \left(-\frac{\pi}{2}-\Theta_{c}\right) & 0 & -\sin \left(-\frac{\pi}{2}-\Theta_{c}\right) \\
0 & 1 & 0 \\
\sin \left(-\frac{\pi}{2}-\Theta_{c}\right) & 0 & \cos \left(-\frac{\pi}{2}-\Theta_{c}\right)
\end{array}\right], \\
C_{\omega_{c}}=\left[\begin{array}{ccc}
\cos \left(\omega_{c}\right) & \sin \left(\omega_{c}\right) & 0 \\
-\sin \left(\omega_{c}\right) & \cos \left(\omega_{c}\right) & 0 \\
0 & 0 & 1
\end{array}\right] \\
C_{i_{c}}=\left[\begin{array}{ccc}
1 & 0 & 0 \\
0 & \cos \left(i_{c}\right) & \sin \left(i_{c}\right) \\
0 & -\sin \left(i_{c}\right) & \cos \left(i_{c}\right)
\end{array}\right] \\
C_{\Omega_{c}}=\left[\begin{array}{ccc}
\cos \left(\Omega_{c}\right) & \sin \left(\Omega_{c}\right) & 0 \\
-\sin \left(\Omega_{c}\right) & \cos \left(\Omega_{c}\right) & 0 \\
0 & 0 & 1
\end{array}\right] \\
z=\left[\begin{array}{lll}
0 & 0 & 1
\end{array}\right]^{\mathrm{T}}
\end{gathered}
$$

Further details can be found in [29].

\subsection{Graph}

An undirected graph $\mathcal{G}(t)$ on a fixed node set is a pair $(\mathcal{N}, \varepsilon, \mathcal{C}(t))$, where $\mathcal{N}=\{1 \ldots n\}$ is the node set, $\varepsilon \subseteq \mathcal{N} \times \mathcal{N}$ implies the edge set, and $\mathcal{C}(t) \in R^{n \times n}$ denotes the weight matrix. The arc $(i, j) \in \varepsilon$ if and only if node $i$ transmits information to node $j$ and vice versa. For each node $i \in \mathcal{N}$, the number of connected arc $(i, j) \in \varepsilon$ is the out-degree, denoted by $d(i)$. A path in an undirected graph is a sequence of edges joining a sequence of distinct nodes. For any pair of nodes $i, j \in \mathcal{N}$, if there exists a path joining $i$ and $j$, the undirected graph is connected. In the formalism of weighted undirected graphs, every edge $(i, j)$ has a weight coefficient $[\mathcal{C}(t)]_{i, j} \in \boldsymbol{R}_{\geq 0}$, and the matrix $\mathcal{C}(t)$ is also called adjacency matrix of graph $\mathcal{G}(t)$. The Laplacian matrix of graph $\mathcal{G}(t)$ is denoted by $\boldsymbol{L}_{A}(t) \in \boldsymbol{R}^{n \times n}$ and it is defined through its elements

$$
\left[\boldsymbol{L}_{A}(t)\right]_{i, j}=\left\{\begin{array}{cc}
-[\mathcal{C}(t)]_{i, j} & \text { if } i \neq j \\
\sum_{j=1, j \neq i}^{n}[\mathcal{C}(t)]_{i, j} & \text { else }
\end{array} .\right.
$$

We present here three important results that have been proposed in [18]

Proposition 1. Given $\forall i=1 \ldots n, \boldsymbol{X}_{i} \in \boldsymbol{R}^{p}$ and $\boldsymbol{X}=\left[\boldsymbol{X}_{1}^{\mathrm{T}} \ldots \boldsymbol{X}_{n}^{\mathrm{T}}\right]^{\mathrm{T}}$.

Then, $\forall t \in \boldsymbol{R}_{\geq 0}, \boldsymbol{X}^{\mathrm{T}}\left(\boldsymbol{L}_{A}(t) \otimes \mathbf{I}_{p}\right) \boldsymbol{X}=\frac{1}{2} \sum_{i=1}^{n} \sum_{j=1}^{n}[\mathcal{C}(t)]_{i, j}(t)\left\|\boldsymbol{X}_{i}-\boldsymbol{X}_{j}\right\|^{2}$.

Proposition 2. If undirected $\mathcal{G}(t)$ is connected, $\left(\boldsymbol{L}_{A}(t) \otimes \boldsymbol{I}_{p}\right) \boldsymbol{X}=\mathbf{0}_{n p \times 1} \Leftarrow \forall i \neq j, \boldsymbol{X}_{i}=\boldsymbol{X}_{j}$.

Proposition 3. The Laplacian matrix $\mathbf{L}_{A}(t)$ of an undirected graph is positive semidefinite.

\subsection{The Model for Wireless Interference}

In the wireless communication scheme of spacecraft swarm, physically, when multiple electromagnetic waves are broadcast by a set of spacecraft in the same frequency band and superimposed at the receiver, it results in interference [31]. Theoretically, interference can be utilized to allow multiple spacecraft to access the same channel frequency spectrum simultaneously. As a result, if interference is utilized in the communication scheme, the communication resources can be saved proportionally to the number of spacecraft com- 
pared to OCAM. It should be noted that the communication system exploiting interference suffers from unknown fading channels, which is a challenge that should be dealt with.

A wireless multiple access channel (WMAC) allows modeling the value at the receiver in the wireless communication scheme, which exploits interference [4,32]. Let $\boldsymbol{\Gamma}_{i} \in \boldsymbol{R}^{p}$ be the real-valued vector signal broadcast by the spacecraft $i, i \in 1 \ldots n$ and $\mathbf{Y} \in \boldsymbol{R}^{p}$ be the value at the receiver, then the mapping: $\mathbf{Y}=\sum_{i=1 \ldots n} \mathcal{C}_{i, r} \Gamma_{i}$ is referred to as the WMAC, where $\mathcal{C}_{i, r} \in \boldsymbol{R}$ is the unknown fading coefficient of transmitter $i$.

The way interference can be exploited, and the unknown fading coefficient can be handled for the spacecraft swarm at hand will be clear in Section 3.2, where a communication system compatible with the WMAC will be presented.

\subsection{Stabilization Theory of Sampled-Data Nonlinear Systems}

Consider the system

$$
\dot{x}=g(\boldsymbol{x}, \boldsymbol{u}),
$$

where $\boldsymbol{X} \in \boldsymbol{R}^{p}, \boldsymbol{u} \in \boldsymbol{R}^{n}$ and $g(\boldsymbol{x}, \boldsymbol{u})$ are locally Lipschitz. Given constant sampling intervals $\left[t_{k}, t_{k+1}\right), \forall t_{k} \in \boldsymbol{R}_{\geq 0}, k \in \boldsymbol{N}_{\geq 0}$, with $\Delta t=t_{k+1}-t_{k}$, the control $\boldsymbol{u}$ is updated at sampling instants, i.e., during the period $\left[t_{k}, t_{k+1}\right), \boldsymbol{u}(t)=\boldsymbol{u}(k)$. The exact discrete-time model of (4) is

$$
x(k+1)=G^{e}(x(k), u(k)),
$$

which is the closed-form solution to the initial value problem

$$
\dot{x}=g(x, u(k)), \quad x_{0}=x(k),
$$

over the sampling interval $\left[t_{k}, t_{k+1}\right)$, which is impossible to obtain in general. However, the following Euler's first-order approximation is available

$$
x(k+1)=G^{\text {Euler }}(x(k), \boldsymbol{u}(k))=x(k)+\Delta t g(x(k), \boldsymbol{u}(k)) .
$$

Then, the following Lemmas are introduced $[33,34]$

Lemma 1. If there exist $f(\cdot) \in \mathcal{K}_{\infty}$ and $M \in \boldsymbol{R}_{>0}, T^{*} \in \boldsymbol{R}_{>0}$, for all $\Delta t \in\left[0, T^{*}\right]$, and $X, Y \in R^{n}:$

1. $|g(\boldsymbol{x}, \boldsymbol{u}(k))| \leq M$,

2. $|g(\boldsymbol{y}, \boldsymbol{u}(k))-| g(\boldsymbol{x}, \boldsymbol{u}(k)) \mid \leq f(|\boldsymbol{y}-\boldsymbol{x}|)$,

Then $G^{\text {Euler }}(\boldsymbol{x}(k), \boldsymbol{u}(k))$ is one-step consistent with $G^{e}(\boldsymbol{x}(k), \boldsymbol{u}(k))$.

Lemma 2. Let $\beta \in \mathcal{K}_{\mathfrak{w}}$ and let $\boldsymbol{A} \subset \boldsymbol{R}^{n}$ be an open (not necessarily bounded) set containing the origin. The family $(\boldsymbol{u}(k), G(\boldsymbol{x}(k), \boldsymbol{u}(k)))$ is said to be $(\beta, \boldsymbol{A})$-practically stable if $\forall M \in \boldsymbol{R}_{>0}$ there exists $T^{*}>0$ such that for each $\Delta t \in\left[0, T^{*}\right]$, the solutions of the system

$$
\boldsymbol{x}(k+1)=G(x(k), \boldsymbol{u}(k)),
$$

satisfy

$$
|x(k, x(0))| \leq \beta(|x(0)|, \mathrm{k} \Delta t)+M, \forall x(0) \in A, k \in N_{\geq 0} .
$$

Lemma 3. The family $(\boldsymbol{u}(k), G(\boldsymbol{x}(k), \boldsymbol{u}(k)))$ is said to be equi-globally asymptotically stable (EGAS) if the following statements hold

1. There exist $T^{*}>0, \alpha_{1}(\cdot), \alpha_{2}(\cdot) \in \mathcal{K}_{\infty}, \alpha_{3}(\cdot) \in \mathcal{K}$ and for each $\Delta t \in\left[0, T^{*}\right], V(\cdot): \boldsymbol{R}^{n} \rightarrow \boldsymbol{R}_{\geq 0}$ such that $\forall \boldsymbol{X} \subset \boldsymbol{R}^{n}, \forall \Delta t \in\left[0, T^{*}\right]$, we have $\alpha_{1}(|\boldsymbol{X}|) \leq V(\boldsymbol{X}) \leq \alpha_{2}(|\boldsymbol{X}|)$ and $V(G(\boldsymbol{X}, \boldsymbol{u}(\boldsymbol{X})))-$ $V(\boldsymbol{X}) \leq-\Delta t \alpha_{3}(|\boldsymbol{X}|)$.

2. For each compact set $Y \subset \boldsymbol{R}^{n} \backslash \mathbf{0}$, there exist $M>0$ and $T^{*}>0$ such that, $\forall \boldsymbol{A}, \boldsymbol{B} \in \mathbf{Y}$ and $\forall \Delta t \in\left[0, T^{*}\right],|V(\boldsymbol{A})-V(\boldsymbol{B})| \leq M|\boldsymbol{A}-\boldsymbol{B}|$. 
Lemma 4. If $G^{\text {Euler }}(\boldsymbol{x}(k), \boldsymbol{u}(k))$ is EGAS, then there exists $\beta \in \mathcal{K} \mathfrak{w}$ such that for each bounded neighborhood $A$ of the origin, the exact discrete-time models $G^{e}(x(k), u(k))$ in Equation (5) is $(\beta, A)$-practically stable.

\subsection{The Mathematic Model of Problem Description}

Suppose each spacecraft in the swarm is an agent modeled by (2) and (3). The orbit elements of the spacecraft in the swarm are predesigned. The orbit elements of the moving target can only be obtained by the LS. To track the moving target, the following two objects need to be achieved.

Object 1. The attitude $Q_{1}$ of the LS is calculated according to the orbit elements of the moving target and the orbit elements of itself, details will be given in Section 3.2.

Object 2. The whole swarm needs to achieve attitude synchronization with the LS, namely, $Q_{i}=Q_{1}, i=2 \ldots n$.

It is worth mentioning that according to Equation (1), the Object 2 turns into spacecraft attitude cooperative tracking problem $\boldsymbol{Q}_{k, i}=\boldsymbol{Q}_{1}, i=2 \ldots n$, where $\boldsymbol{Q}_{k, i}=[\overbrace{\boldsymbol{Q}_{m, i}^{\times}}^{\times}] \boldsymbol{Q}_{i}, \boldsymbol{Q}_{m, i}$ is preset desired attitude difference between $Q_{i}, i=2 \ldots n$ and $Q_{1}$. In other words, the method in this paper is applicable to typical spacecraft attitude cooperative tracking problem.

Interference is used for information exchange in this paper to allow multiple spacecraft to access the same channel frequency spectrum simultaneously, and the technical detail can be found in Section 3.2. It should be noted that when exploiting interference, the unknown fading channels in the radio frequency $(\mathrm{RF})$ communication system constitutes a challenge that the control law needs to deal with.

The communication topology in this paper is modeled as undirected connected graph $\mathcal{G}(t)=(\mathcal{N}, \varepsilon, \mathcal{C}(t))$. Throughout this paper, graph $\mathcal{G}(t)$ always satisfies the following assumption

Assumption 1. Graph $\mathcal{G}(t)$ is an undirected connected graph, hence for any pair of connected nodes $i, j \in \mathcal{N}$, there must be $[\mathcal{C}(t)]_{i, j}=[\mathcal{C}(t)]_{j, i}$.

The communication scheme is discrete in time. The spacecraft update state at every instant $t_{k} \in \boldsymbol{R}_{\geq 0}, \quad k \in \boldsymbol{N}_{\geq 0}$, and the interval between any two adjacent update instants is defined as $\Delta t \in \boldsymbol{R}_{>0}$.

\section{Control Law Design}

At first, we calculate the attitude of the LS according to the orbit elements of the target and the orbit elements of the LS. After that, a communication system exploiting interference (the value at the receiver modeled by the WMAC) is proposed. Based on the communication scheme, we finally provide the discrete control approach for all follower spacecraft (FS).

The following lemmas will be used in the subsequent control law design:

Lemma 5. A symmetric matrix $A \in \boldsymbol{R}^{n \times n}$ with eigenvalues $C_{1} \ldots C_{n}$ is orthogonally diagonalizable, and can be decomposed as [35]

$$
A=V C V^{T}
$$

where $\boldsymbol{C}=\operatorname{diag}\left(C_{1} \ldots C_{n}\right), \boldsymbol{V} \in \boldsymbol{R}^{n \times n}$ is an orthogonal matrix whose columns correspond to the eigenvectors of $A$. Additionally, we also have

$$
V^{T} A V=C
$$


such that matrix $A$ is orthogonally similar to a diagonal matrix formed from the eigenvalues of $\boldsymbol{A}$.

Lemma 6. A matrix $V \in \boldsymbol{R}^{n \times n}$ is an orthogonal matrix if and only if its column (row) vector group is a group of standard orthogonal base of $\boldsymbol{R}^{n}$ [35].

Lemma 7. Given a matrix $\boldsymbol{D}: \boldsymbol{D}=\left[\begin{array}{cc}\boldsymbol{A} & \boldsymbol{B} \\ \boldsymbol{B}^{\mathrm{T}} & \boldsymbol{C}\end{array}\right]$, is a positive definite matrix if and only if $\boldsymbol{A} \in$ $\boldsymbol{R}^{r \times r}$ and $\boldsymbol{C} \in \boldsymbol{R}^{(n-r) \times(n-r)}$ are positive definite, and $\boldsymbol{C}-\boldsymbol{B}^{\mathrm{T}} \boldsymbol{A}^{-1} \boldsymbol{B}$ is also positive definite [36].

Lemma 8. For real symmetric matrix $\boldsymbol{A}, \boldsymbol{B} \in \boldsymbol{R}^{n \times n}$, where $\boldsymbol{B}=\operatorname{diag}\left(k_{1}, \ldots, k_{n}\right)$, the following inequality holds

$$
\lambda_{j}(\boldsymbol{A})+\min \left(k_{i} \mid i=1 \ldots n\right) \leq \lambda_{j}(\boldsymbol{A}+\boldsymbol{B}) \leq \lambda_{j}(\boldsymbol{A})+\max \left(k_{i} \mid i=1 \ldots n\right),
$$

where for any matrix $\mathbf{Z} \in \boldsymbol{R}^{n \times n}$, the eigenvalues are arranged as $\lambda_{1}(\mathbf{Z}) \leq \ldots \leq \lambda_{n}(\mathbf{Z})$.

Proof of Lemma 8. Given a real matrix $K=k \mathbf{I}_{n}, k \in\left\{k_{i} \mid i=1 \ldots n\right\}$, according to Lemma 5 ,

$$
\boldsymbol{A}=\boldsymbol{V} \boldsymbol{C} \boldsymbol{V}^{\mathrm{T}}
$$

where $C=\operatorname{diag}\left(C_{1} \ldots C_{n}\right), V \in \boldsymbol{R}^{n \times n}$ is an orthogonal matrix whose columns correspond to the eigenvectors of $A$.

Hence

$$
\begin{aligned}
\boldsymbol{A}+\boldsymbol{K} & =\boldsymbol{A}+k \mathbf{I}_{n} \\
& =\boldsymbol{V} \boldsymbol{C} \boldsymbol{V}^{\mathrm{T}}+k \boldsymbol{V} \boldsymbol{V}^{\mathrm{T}} \\
& =\boldsymbol{V} \operatorname{diag}\left(C_{1}+k \ldots C_{n}+k\right) \boldsymbol{V}^{\mathrm{T}},
\end{aligned}
$$

then $\lambda(\boldsymbol{A}+\boldsymbol{K})=\lambda(\boldsymbol{A})+k$ is obtained.

According to $A .1 . b$ in [37] (p. 510), as

$$
\left(\boldsymbol{A}+\max \left(k_{i} \mid i=1 \ldots n\right) \mathbf{I}_{n}\right)-(\boldsymbol{A}+\boldsymbol{B}) \geq 0,
$$

the following inequality is obtained

$$
\lambda_{j}(\boldsymbol{A}+\boldsymbol{B}) \leq \lambda_{j}(\boldsymbol{A})+\max \left(k_{i} \mid i=1 \ldots n\right), j=1 \ldots n .
$$

In the same way, we get the following inequality

$$
\lambda_{j}(\boldsymbol{A})+\min \left(k_{i} \mid i=1 \ldots n\right) \leq \lambda_{j}(\boldsymbol{A}+\boldsymbol{B})
$$

Lemma 9. For real positive semidefinite matrix $\boldsymbol{A} \in \boldsymbol{R}^{n \times n}$ and $\boldsymbol{B} \in \boldsymbol{R}^{n \times n}$, the following property holds

$$
\lambda_{\max }(\boldsymbol{A B}) \leq \lambda_{\max }(\boldsymbol{A}) \lambda_{\max }(\boldsymbol{B})
$$

Proof of Lemma 9. According to Corollary 11 in [38], for the Hermitian matrix $\boldsymbol{A} \in \boldsymbol{R}^{n \times n}$, real positive semidefinite matrix $\boldsymbol{B} \in \boldsymbol{R}^{n \times n}$, and $\boldsymbol{U} \in \boldsymbol{R}^{1 \times n}$ satisfying $\boldsymbol{U} \boldsymbol{U}^{\mathrm{T}}=1$, the inequality $\lambda_{\max }(\boldsymbol{A B}) \leq \max _{\boldsymbol{U} \boldsymbol{U}^{\mathrm{T}}=1} \lambda_{\max }(\boldsymbol{A}) \lambda_{\max }\left(\boldsymbol{U} \boldsymbol{B} \boldsymbol{U}^{\mathrm{T}}\right)$ holds. Then according to Equation (1) in [37], we have the equality $\max _{\boldsymbol{U} \boldsymbol{U}^{\mathrm{T}}=1}\left(\boldsymbol{U} \boldsymbol{B} \boldsymbol{U}^{\mathrm{T}}\right)=\lambda_{\max }(\boldsymbol{B})$. When $\lambda_{\max }(\boldsymbol{A})>0$, the Lemma 9 is obtained. 
Lemma 10. For real symmetric matrix $\boldsymbol{A} \in \boldsymbol{R}^{n \times n}$ with eigenvalues $\lambda_{1} \geq \ldots \geq \lambda_{n}$, and $\boldsymbol{U} \in \boldsymbol{R}^{k \times n}$ $U U^{\mathrm{T}}=I_{k}$, we get the following inequality

$$
\lambda_{\max }\left(U A \boldsymbol{U}^{\mathrm{T}}\right) \leq \lambda_{1}
$$

Proof of Lemma 10. According to Lemma 5,

$$
\boldsymbol{A}=\boldsymbol{V C} \boldsymbol{V}^{\mathrm{T}}
$$

where $C=\operatorname{diag}\left(\lambda_{1} \ldots \lambda_{n}\right), V \in \boldsymbol{R}^{n \times n}$ is an orthogonal matrix whose columns correspond to the eigenvectors of $A$. Hence

$$
U A U^{\mathrm{T}}=U \boldsymbol{U C V} \boldsymbol{V}^{\mathrm{T}} \boldsymbol{U}^{\mathrm{T}}
$$

define

$$
U V=W,
$$

then

$$
\mathbf{W} \boldsymbol{W}^{\mathrm{T}}=\mathbf{I}_{k}
$$

is derived. Additionally, $U A U^{\mathrm{T}}$ can be rewritten as

$$
U A U^{\mathrm{T}}=W C \boldsymbol{W}^{\mathrm{T}} .
$$

Define $C_{\text {max }}=\operatorname{diag}\left(\lambda_{1} \ldots \lambda_{1}\right) \in \boldsymbol{R}^{n \times n}$, then

$$
\boldsymbol{W C} \boldsymbol{W}^{\mathrm{T}}-\boldsymbol{W C}_{\max } \boldsymbol{W}^{\mathrm{T}}=\boldsymbol{W}\left(\boldsymbol{C}-\boldsymbol{C}_{\max }\right) \boldsymbol{W}^{\mathrm{T}} \leq 0_{k \times k},
$$

which means

$$
U A \boldsymbol{U}^{\mathrm{T}} \leq \boldsymbol{W} \boldsymbol{C}_{\max } \boldsymbol{W}^{\mathrm{T}},
$$

where $\boldsymbol{W C}_{\text {max }} \boldsymbol{W}^{\mathrm{T}}=\operatorname{diag}\left(\lambda_{1} \ldots \lambda_{1}\right) \in \boldsymbol{R}^{k \times k}$.

According to $A .1 . b$ in [37] (p. 510), $\lambda_{\max }\left(U A \boldsymbol{U}^{\mathrm{T}}\right) \leq \lambda_{1}$ is proved.

\subsection{Attitude Determination of the Virtual Leader Spacecraft}

First of all, the position vector $\boldsymbol{P}_{t}=\left[P_{x, t}, P_{y, t}, P_{z, t}\right]^{\mathrm{T}} \in \boldsymbol{R}^{3}$ of the target and the position vector $\boldsymbol{P}_{s}=\left[P_{x, s}, P_{y, s}, P_{z, s}\right]^{\mathrm{T}} \in \boldsymbol{R}^{3}$ of the virtual leader spacecraft in $O_{\mathrm{I}} X_{\mathrm{I}} Y_{\mathrm{I}} Z_{\mathrm{I}}$ is obtained by the orbit elements according to the process in Section 2.1.4. Then the unit vectors $X_{\mathrm{BI}}, Y_{\mathrm{BI}}$, and $Z_{\mathrm{BI}}$ along the positive direction of $\mathrm{O}_{\mathrm{B}} X_{\mathrm{B}}, \mathrm{O}_{\mathrm{B}} Y_{\mathrm{B}}$, and $\mathrm{O}_{\mathrm{B}} Z_{\mathrm{B}}$ axes are

$$
\begin{aligned}
& X_{\mathrm{BI}}=C_{\mathrm{BI}} x, \\
& Y_{\mathrm{BI}}=C_{\mathrm{BI}} y, \\
& Z_{\mathrm{BI}}=C_{\mathrm{BI}} z,
\end{aligned}
$$

where $x=\left[\begin{array}{lll}1 & 0 & 0\end{array}\right]^{\mathrm{T}}, \boldsymbol{y}=\left[\begin{array}{lll}0 & 1 & 0\end{array}\right]^{\mathrm{T}}$ and $z=\left[\begin{array}{lll}0 & 0 & 1\end{array}\right]^{\mathrm{T}}$. Furthermore, the vector from the virtual leader spacecraft to the target is $\boldsymbol{P}_{s t}=\boldsymbol{P}_{t}-\boldsymbol{P}_{s}$. Hence the unit vectors $X_{\mathrm{DI}}, Y_{\mathrm{DI}}$, and $Z_{\mathrm{DI}}$ along the positive direction of $O_{\mathrm{D}} X_{\mathrm{D}}, O_{\mathrm{D}} Y_{\mathrm{D}}$ and $O_{\mathrm{D}} Z_{\mathrm{D}}$ axes are

$$
\begin{gathered}
Z_{\mathrm{DI}}=\frac{\boldsymbol{P}_{s t}}{\left|\boldsymbol{P}_{s t}\right|}, \\
X_{\mathrm{DI}}=\frac{Z_{\mathrm{DI}} \times\left(-\boldsymbol{Y}_{\mathrm{BI}}\right)}{\left|\mathbf{Z}_{\mathrm{DI}} \times\left(-\boldsymbol{Y}_{\mathrm{BI}}\right)\right|}, \\
Y_{\mathrm{DI}}=\frac{Z_{\mathrm{DI}} \times \boldsymbol{X}_{\mathrm{DI}}}{\left|\mathbf{Z}_{\mathrm{DI}} \times \boldsymbol{X}_{\mathrm{DI}}\right|} .
\end{gathered}
$$


Finally, referring to the Euler angle attitude representation in [29], the desired attitude, i.e., the attitude of the virtual leader represented by Euler angle $\left[\begin{array}{lll}\phi_{1} & \theta_{1} & \psi_{1}\end{array}\right]^{\mathrm{T}}$ is calculated by

$$
\begin{gathered}
\phi_{1}=\tan ^{-1}\left(\frac{\boldsymbol{Y}_{\mathrm{DI}} \cdot \boldsymbol{Z}_{\mathrm{BI}}}{\left|\boldsymbol{Z}_{\mathrm{DI}} \cdot \boldsymbol{Z}_{\mathrm{BI}}\right|}\right), \\
\theta_{1}=\sin ^{-1}\left(-\boldsymbol{X}_{\mathrm{DI}} \cdot \boldsymbol{Z}_{\mathrm{BI}}\right), \\
\psi_{1}=\tan ^{-1}\left(\frac{\boldsymbol{X}_{\mathrm{DI}} \cdot \boldsymbol{Y}_{\mathrm{BI}}}{\left|\boldsymbol{X}_{\mathrm{DI}} \cdot \boldsymbol{X}_{\mathrm{BI}}\right|}\right) .
\end{gathered}
$$

The quaternion $Q_{1}=\left[q_{0,1}, q_{1,1}, q_{2,1}, q_{3,1}\right]^{\mathrm{T}}$ corresponding to $\left[\begin{array}{lll}\phi_{1} & \theta_{1} & \psi_{1}\end{array}\right]^{\mathrm{T}}$ is expressed as

$$
\begin{gathered}
q_{0,1}=\cos \left(\frac{\phi_{1}}{2}\right) \cos \left(\frac{\theta_{1}}{2}\right) \cos \left(\frac{\psi_{1}}{2}\right)+\sin \left(\frac{\phi_{1}}{2}\right) \sin \left(\frac{\theta_{1}}{2}\right) \sin \left(\frac{\psi_{1}}{2}\right), \\
q_{1,1}=\sin \left(\frac{\phi_{1}}{2}\right) \cos \left(\frac{\theta_{1}}{2}\right) \cos \left(\frac{\psi_{1}}{2}\right)-\cos \left(\frac{\phi_{1}}{2}\right) \sin \left(\frac{\theta_{1}}{2}\right) \sin \left(\frac{\psi_{1}}{2}\right), \\
q_{2,1}=\cos \left(\frac{\phi_{1}}{2}\right) \sin \left(\frac{\theta_{1}}{2}\right) \cos \left(\frac{\psi_{1}}{2}\right)+\sin \left(\frac{\phi_{1}}{2}\right) \cos \left(\frac{\theta_{1}}{2}\right) \sin \left(\frac{\psi_{1}}{2}\right), \\
q_{3,1}=-\sin \left(\frac{\phi_{1}}{2}\right) \sin \left(\frac{\theta_{1}}{2}\right) \cos \left(\frac{\psi_{1}}{2}\right)+\cos \left(\frac{\phi_{1}}{2}\right) \cos \left(\frac{\theta_{1}}{2}\right) \sin \left(\frac{\psi_{1}}{2}\right) .
\end{gathered}
$$

\subsection{Communication System Exploiting Interference}

For each spacecraft $i$ in the swarm, the three broadcasting states from every neighbor $j,(j, i) \in \varepsilon$ at consecutive broadcasting instants $t_{k} \in \boldsymbol{R}_{\geq 0}, k \in \boldsymbol{N}_{\geq 0}$ is designed according to $[39]$ as

$$
\begin{aligned}
\boldsymbol{\mu}_{j}^{(1)}(k) & =\boldsymbol{Q}_{j}(k), \\
\boldsymbol{\mu}_{j}^{(2)}(k) & =\boldsymbol{X}_{1 \mathrm{~F}, j}(k), \\
\boldsymbol{\mu}_{j}^{\prime}(k) & =1,
\end{aligned}
$$

where $Q_{j}$ is attitude quaternion of spacecraft $j, X_{1 \mathrm{~F}, j}$ is a state of spacecraft $j$ and will be introduced in Section 3.3. So at update instants $t_{k}$, the spacecraft $i$ receives three signals

$$
\begin{aligned}
\boldsymbol{\tau}_{i}^{(1)}(k) & =\sum_{(j, i) \in \varepsilon}[\mathcal{C}(t)]_{j, i}(k) \mathbf{Q}_{j}(k), \\
\boldsymbol{\tau}_{i}^{(2)}(k) & =\sum_{(j, i) \in \varepsilon}[\mathcal{C}(t)]_{j, i}(k) \boldsymbol{X}_{1 \mathrm{~F}, j}(k), \\
\boldsymbol{\tau}_{i}^{\prime}(k) & =\sum_{(j, i) \in \varepsilon}[\mathcal{C}(t)]_{j, i}(k),
\end{aligned}
$$

where $[\mathcal{C}(t)]_{j, i}(k)$ is the unknown fading coefficient of the edge $(j, i) \in \varepsilon$ at broadcasting instants $t_{k}$. Then the signals $\tau_{i}^{(1)}(k)$ and $\tau_{i}^{(2)}(k)$ in Equation (14) are normalized, hence the signals received by spacecraft $i$ are converted to

$$
\begin{aligned}
\boldsymbol{\zeta}_{i}^{(1)}(k) & =\left[\sum_{(j, i) \in \varepsilon}[\mathcal{C}(t)]_{j, i}(k) \mathbf{I}_{4}\right]^{-1} \sum_{(j, i) \in \mathcal{\varepsilon}}[\mathcal{C}(t)]_{j, i}(k) Q_{j}(k), \\
\zeta_{i}^{(2)}(k) & =\left[\sum_{(j, i) \in \varepsilon}[\mathcal{C}(t)]_{j, i}(k) \mathbf{I}_{4}\right]^{-1} \sum_{(j, i) \in \varepsilon}[\mathcal{C}(t)]_{j, i}(k) X_{1 \mathrm{~F}, j}(k) .
\end{aligned}
$$


Obviously, by exploiting interference, the signals each spacecraft received are the sum of the relevant signals transmitted by their neighbors. Instead of using OCAM to receive accurate signals transmitted by the neighbors, the communication system utilizing interference can save the communication resources proportionally to the number of spacecraft.

3.3. Design of Attitude Tracking Synchronization Control Scheme

3.3.1. Continuous Control Scheme

Define

$$
\boldsymbol{Q}=\left[\boldsymbol{Q}_{1}^{\mathrm{T}} \ldots \boldsymbol{Q}_{n}^{\mathrm{T}}\right]^{\mathrm{T}} \in \boldsymbol{R}^{4 n}, \boldsymbol{\omega}=\left[\boldsymbol{\omega}_{1}^{\mathrm{T}} \ldots \boldsymbol{\omega}_{n}^{\mathrm{T}}\right]^{\mathrm{T}} \in \boldsymbol{R}^{3 n},
$$

and

$$
\boldsymbol{J}=\operatorname{diag}\left(\boldsymbol{J}_{1} \ldots \boldsymbol{J}_{n}\right),
$$

where $J_{1}$ is the momentum of inertia of the LS, and $J_{i}, i=2 \cdots n$ are the momentum of inertia of the FS. Inspired by [18], define

$$
\widetilde{\boldsymbol{Q}}=\left(\boldsymbol{L}_{A}(t) \otimes \mathbf{I}_{4}\right)^{\mathrm{T}} \boldsymbol{Q} \in \boldsymbol{R}^{4 n},
$$

which is a column stack vector of all

$$
\sum_{j=1}^{n}\left([\mathcal{C}(t)]_{j, i}(t)\left(Q_{i}-Q_{j}\right)\right), i=1 \cdots n .
$$

Define the first backstepping state $X_{1 \mathrm{~F}} \in \boldsymbol{R}^{4 n}$ as

$$
\begin{aligned}
& \boldsymbol{X}_{1 \mathrm{~F}}=\operatorname{diag}\left(\mathbf{I}_{4} \boldsymbol{\zeta}_{1}^{\prime}(t), \ldots, \mathbf{I}_{4} \boldsymbol{\zeta}_{n}^{\prime}(t)\right)^{-1} \widetilde{\boldsymbol{Q}} \\
& =\left[\begin{array}{c}
\frac{\sum_{(j, 1) \in \varepsilon}\left([\mathcal{C}(t)]_{j, 1}(t)\left(Q_{1}-Q_{j}\right)\right)}{\sum_{(j, 1) \in \varepsilon}[\mathcal{C}(t)]_{j, 1}(t)} \\
\vdots \\
\frac{\sum_{(j, n) \in \varepsilon}\left([\mathcal{C}(t)]_{j, n}(t)\left(Q_{n}-Q_{j}\right)\right)}{\left.\sum_{(j, n) \in \varepsilon} \mathcal{C}(t)\right]_{j, n}(t)}
\end{array}\right] \\
& =\left[\begin{array}{c}
\frac{\left(\sum_{(j, 1) \in \varepsilon}[\mathcal{C}(t)]_{j, 1}(t)\right) Q_{1}-\sum_{(j, 1) \in \varepsilon}\left(c_{j, 1}(t)\right.}{\sum_{(j, 1) \in \varepsilon}[\mathcal{C}(t)]_{j, 1}(t)} \\
\vdots \\
\frac{\left(\sum_{(j, n) \in \varepsilon}[\mathcal{C}(t)]_{j, n}(t)\right) Q_{n}-\sum_{(j, n) \in \varepsilon}\left([\mathcal{C}(t)]_{j, n}(t) Q_{j}\right)}{\sum_{(j, n) \in \varepsilon}[\mathcal{C}(t)]_{j, n}(t)}
\end{array}\right] \\
& =\left[\begin{array}{c}
Q_{1}-\varsigma_{1}^{(1)}(t) \\
\vdots \\
Q_{n}-\boldsymbol{\zeta}_{n}^{(1)}(t)
\end{array}\right], \\
& \boldsymbol{X}_{1 \mathrm{~F}, i}=\left[\begin{array}{c}
{\left[\boldsymbol{X}_{1 \mathrm{~F}}\right]_{4 i-3,1}} \\
{\left[\boldsymbol{X}_{1 \mathrm{~F}}\right]_{4 i-2,1}} \\
{\left[\boldsymbol{X}_{1 \mathrm{~F}}\right]_{4 i-1,1}} \\
{\left[\boldsymbol{X}_{1 \mathrm{~F}}\right]_{4 i, 1}}
\end{array}\right], i=1 \ldots n .
\end{aligned}
$$

When the attitudes of all spacecraft in the swarm realize synchronization, that is $\widetilde{Q}=\mathbf{0}_{4 n \times 1}$, we have $\boldsymbol{X}_{1 \mathrm{~F}}=\mathbf{0}_{4 n \times 1}$. A virtual control input is defined as

$$
\boldsymbol{\omega}=f_{n}\left(\boldsymbol{X}_{\mathrm{AF}}\right)+\boldsymbol{X}_{2 \mathrm{~F}}
$$


where $f_{n}\left(\boldsymbol{X}_{\mathrm{AF}}\right) \in \boldsymbol{R}^{3 n}$ is a stabilizing function and $\boldsymbol{X}_{2 \mathrm{~F}} \in \boldsymbol{R}^{3 n}$ is the second backstepping state. Considering all the spacecraft receiving their neighbors' data only at update instants $t_{k} \in \boldsymbol{R}_{\geq 0}, k \in \boldsymbol{N}_{\geq 0}$, during the period $\forall\left[t_{k}, t_{k+1}\right)$, the $\boldsymbol{X}_{1 \mathrm{~F}}$-dynamics is given by

$$
\begin{aligned}
\dot{\boldsymbol{X}}_{1 \mathrm{~F}} & =\frac{1}{2} \operatorname{diag}\left(\mathbf{I}_{4} \boldsymbol{\zeta}_{1}^{\prime}\left(t_{k}\right), \ldots, \mathbf{I}_{4} \boldsymbol{\zeta}_{n}^{\prime}\left(t_{k}\right)\right)^{-1}\left(\boldsymbol{L}_{A}\left(t_{k}\right) \otimes \mathbf{I}_{4}\right)^{\mathrm{T}} \times \\
& \operatorname{diag}\left(\Xi\left[\boldsymbol{Q}_{1}\right] \ldots \Xi\left[\boldsymbol{Q}_{n}\right]\right) \boldsymbol{\omega} \\
& =\frac{1}{2} \mathcal{L}^{\mathrm{T}}\left(t_{k}\right) \boldsymbol{P}_{2}^{\mathrm{T}} \boldsymbol{\omega},
\end{aligned}
$$

where

$$
\mathcal{L}^{\mathrm{T}}=\mathcal{L}=\operatorname{diag}\left(\mathbf{I}_{4} \boldsymbol{\zeta}_{1}^{\prime}, \ldots, \mathbf{I}_{4} \boldsymbol{\zeta}_{n}^{\prime}\right)^{-1}\left(\boldsymbol{L}_{A} \otimes \mathbf{I}_{4}\right)^{\mathrm{T}}
$$

With some calculations, it can be shown that

$$
\boldsymbol{P}_{2}\left(\boldsymbol{L}_{A} \otimes \mathbf{I}_{4}\right) \operatorname{diag}\left(\mathbf{I}_{4} \boldsymbol{\zeta}_{1}^{\prime}, \ldots, \mathbf{I}_{4} \boldsymbol{\zeta}_{n}^{\prime}\right)^{-1} \boldsymbol{X}_{1 \mathrm{~F}}=\mathbf{0}_{3 n \times 1} \rightarrow \boldsymbol{X}_{1 \mathrm{~F}}=\mathbf{0}_{4 n \times 1},
$$

and $P_{2}$ have the property as follows

$$
\boldsymbol{P}_{2} \boldsymbol{P}_{2}^{\mathrm{T}}=\mathbf{I}_{3 n}
$$

Choose the first candidate Lyapunov function as

$$
\begin{aligned}
V_{1 \mathrm{~F}} & =X_{1 \mathrm{~F}}^{\mathrm{T}} \boldsymbol{X}_{1 \mathrm{~F}} \\
& =\left\|\boldsymbol{Q}_{1}-\boldsymbol{\varsigma}_{1}^{(1)}(t)\right\|^{2}+\ldots+\left\|\boldsymbol{Q}_{n}-\boldsymbol{\zeta}_{n}^{(1)}(t)\right\|_{2} .
\end{aligned}
$$

It thus follows that $V_{1 \mathrm{~F}}$ is positive semidefinite. During the period $\forall\left[t_{k}, t_{k+1}\right)$, the derivative of $V_{1 \mathrm{~F}}$ is given by

$$
\begin{aligned}
\dot{V}_{1 \mathrm{~F}} & =2 \boldsymbol{X}_{1 \mathrm{~F}}^{\mathrm{T}} \dot{\boldsymbol{X}}_{1 \mathrm{~F}} \\
& =\boldsymbol{X}_{1 \mathrm{~F}}^{\mathrm{T}} \mathcal{L}^{\mathrm{T}}\left(t_{k}\right) \boldsymbol{P}_{2}^{\mathrm{T}} \boldsymbol{\omega} \\
& =\boldsymbol{X}_{1 \mathrm{~F}}^{\mathrm{T}} \mathcal{L}^{\mathrm{T}}\left(t_{k}\right) \boldsymbol{P}_{2}^{\mathrm{T}}\left(f_{n}\left(\boldsymbol{X}_{\mathrm{AF}}\right)+\boldsymbol{X}_{2 \mathrm{~F}}\right) \\
& =\boldsymbol{X}_{\mathrm{AF}}^{\mathrm{T}}\left(f_{n}\left(\boldsymbol{X}_{\mathrm{A} F}\right)+\boldsymbol{X}_{2 \mathrm{~F}}\right),
\end{aligned}
$$

where

$$
\begin{aligned}
\boldsymbol{X}_{\mathrm{AF}}=\boldsymbol{P}_{2}\left(\boldsymbol{L}_{A} \otimes \mathbf{I}_{4}\right) \operatorname{diag}\left(\mathbf{I}_{4} \boldsymbol{\zeta}_{1}^{\prime}, \ldots, \mathbf{I}_{4} \boldsymbol{\zeta}_{n}^{\prime}\right)^{-1} \boldsymbol{X}_{1 \mathrm{~F}} \\
=\boldsymbol{P}_{2}\left[\begin{array}{c}
\frac{\sum_{(j, 1) \in \varepsilon}[\mathcal{C}(t)]_{j, 1}(t)\left(\boldsymbol{X}_{1 \mathrm{~F}, 1}-\boldsymbol{X}_{1 \mathrm{~F}, j}\right)}{\sum_{(j, 1) \in \varepsilon}[\mathcal{C}(t)]_{j, 1}(t)} \\
\vdots \\
\frac{\sum_{(j, n) \in \varepsilon}[\mathcal{C}(t)]_{j, n}(t)\left(\boldsymbol{X}_{1 \mathrm{~F}, n}-\boldsymbol{X}_{1 \mathrm{~F}, j}\right)}{\sum_{(j, n) \in \varepsilon}[\mathcal{C}(t)]_{j, n}(t)}
\end{array}\right] \\
=\boldsymbol{P}_{2}\left[\begin{array}{c}
\boldsymbol{Q}_{1}-\boldsymbol{\zeta}_{1}^{(2)}(t) \\
\vdots \\
\boldsymbol{Q}_{n}-\boldsymbol{\zeta}_{n}^{(2)}(t)
\end{array}\right] .
\end{aligned}
$$

Obviously, $f_{n}\left(\boldsymbol{X}_{\mathrm{AF}}\right)$ should satisfy when $\boldsymbol{X}_{1 \mathrm{~F}} \rightarrow \mathbf{0}_{4 n \times 1}$,

$$
\operatorname{diag}\left(\mathbf{I}_{3} \boldsymbol{S}_{1}^{\prime}\left(t_{k}\right), \ldots, \mathbf{I}_{3} \boldsymbol{S}_{n}^{\prime}\left(\left(t_{k}\right)\right)\right)^{-1}\left(\boldsymbol{L}_{A}\left(t_{k}\right) \otimes \mathbf{I}_{3}\right)^{\mathrm{T}} f_{n}\left(\boldsymbol{X}_{\mathrm{AF}}\right) \rightarrow \mathbf{0}_{3 n \times 1},
$$

must be ensured to finally achieve

$$
\operatorname{diag}\left(\mathbf{I}_{3} \boldsymbol{\zeta}_{1}^{\prime}\left(t_{k}\right) \ldots \mathbf{I}_{3} \boldsymbol{\zeta}_{n}^{\prime}\left(\left(t_{k}\right)\right)\right)^{-1}\left(\boldsymbol{L}_{A}\left(t_{k}\right) \otimes \mathbf{I}_{3}\right)^{\mathrm{T}} \boldsymbol{\omega} \rightarrow \mathbf{0}_{3 n \times 1} .
$$


Therefore, $f_{n}\left(\boldsymbol{X}_{\mathrm{AF}}\right)$ can be chosen as

$$
f_{n}\left(\boldsymbol{X}_{\mathrm{A} F}\right)=-\boldsymbol{K}_{1} \boldsymbol{X}_{\mathrm{A} F}
$$

where $\boldsymbol{K}_{1}=\boldsymbol{K}_{1}^{\mathrm{T}}=k_{1} \times \mathbf{I}_{3 \mathrm{n}} \geq \mathbf{0}_{3 \mathrm{n} \times 3 \mathrm{n}}$. Substituting Equation (24) into Equation (22), $\dot{V}_{1 \mathrm{~F}}$ can be written as

$$
\dot{V}_{1 \mathrm{~F}}=\boldsymbol{X}_{\mathrm{A} F}^{\mathrm{T}} \boldsymbol{X}_{2 \mathrm{~F}}-\boldsymbol{X}_{\mathrm{A} F}^{\mathrm{T}} \boldsymbol{K}_{1} \boldsymbol{X}_{\mathrm{A} F} .
$$

From the fact that $K_{1}$ is a positive definite matrix, one arrives $X_{\mathrm{A} F}^{\mathrm{T}} K_{1} X_{\mathrm{A} F}$ is a nonnegative constant. The $\boldsymbol{X}_{1 \mathrm{~F}}$-system in Equation (18) now turns into

$$
\begin{aligned}
\dot{X}_{1 \mathrm{~F}} & =\frac{1}{2} \mathcal{L}^{\mathrm{T}}\left(t_{k}\right) \boldsymbol{P}_{2}^{\mathrm{T}}\left(f_{n}\left(\boldsymbol{X}_{\mathrm{AF}}\right)+\boldsymbol{X}_{2 \mathrm{~F}}\right) \\
& =\frac{1}{2} \mathcal{L}^{\mathrm{T}}\left(t_{k}\right) \boldsymbol{P}_{2}^{\mathrm{T}} \boldsymbol{X}_{2 \mathrm{~F}}-\frac{1}{2} \mathcal{L}^{\mathrm{T}}\left(t_{k}\right) \boldsymbol{P}_{2}^{\mathrm{T}} K_{1} \boldsymbol{X}_{\mathrm{A} F},
\end{aligned}
$$

The $\boldsymbol{X}_{2 \mathrm{~F}}$-dynamics can be obtained by rewriting and differentiating Equation (17) as

$$
\dot{X}_{2 \mathrm{~F}}=\dot{\boldsymbol{\omega}}-\dot{f}_{n}\left(\boldsymbol{X}_{\mathrm{AF}}\right) \text {. }
$$

Substituting Equation (3), Equation (27) can be rewritten as

$$
J \dot{X}_{2 \mathrm{~F}}=\boldsymbol{T}-\boldsymbol{\omega} \times \boldsymbol{J} \boldsymbol{\omega}-\dot{J} \dot{f}_{n}\left(X_{\mathrm{AF}}\right),
$$

where $T \in R^{3 n}$ is a column stack vector of control torque from all the spacecraft's actuators. For the FS, choose the second candidate Lyapunov function as

$$
V_{2 \mathrm{~F}}=V_{1 \mathrm{~F}}+\frac{1}{2} \boldsymbol{X}_{2 \mathrm{~F}}^{\mathrm{T}} \boldsymbol{J} \boldsymbol{X}_{2 \mathrm{~F}}
$$

where $V_{2 \mathrm{~F}}$ is positive semidefinite. Substituting Equation (28), the time derivative of $V_{2 \mathrm{~F}}$ is derived as

$$
\dot{V}_{2 \mathrm{~F}}=\dot{V}_{1 \mathrm{~F}}+\boldsymbol{X}_{2 \mathrm{~F}}^{\mathrm{T}}\left(\boldsymbol{T}-\boldsymbol{\omega} \times \boldsymbol{J} \boldsymbol{\omega}-\boldsymbol{J} \dot{f}_{n}\left(\boldsymbol{X}_{\mathrm{AF}}\right)\right) .
$$

According to Equations (22) and (30), the control torque for the FS can be chosen as

$$
\begin{aligned}
& \boldsymbol{T}=-\boldsymbol{X}_{\mathrm{AF}}-\boldsymbol{K}_{2} \boldsymbol{X}_{2 \mathrm{~F}} \\
&+\boldsymbol{\omega} \times \boldsymbol{J} \boldsymbol{\omega}+\boldsymbol{J} f_{n}\left(\boldsymbol{X}_{\mathrm{AF}}\right), \\
& \boldsymbol{T}_{i}=\left[\begin{array}{c}
{[\boldsymbol{T}]_{3 i-2,1}} \\
{[\boldsymbol{T}]_{3 i-1,1}} \\
{[\boldsymbol{T}]_{3 i, 1}}
\end{array}\right], i=1 \ldots n,
\end{aligned}
$$

where $\boldsymbol{K}_{2}=\boldsymbol{K}_{2}^{\mathrm{T}}=k_{2} \times \mathbf{I}_{3 \mathrm{n}} \geq \mathbf{0}_{3 \mathrm{n} \times 3 \mathrm{n}}$.

One thing that should be mentioned is that $T_{1} \in R^{3}$ is redundant for the FS to achieve the attitude synchronization with the LS. Therefore, $T_{i} \in R^{3}, i=2 \ldots n$ is the input torque for each FS $i$.

Substituting Equation (31) into Equation (30), $\dot{V}_{2 \mathrm{~F}}$ can be written as

$$
\begin{aligned}
\dot{V}_{2 F}= & -\boldsymbol{X}_{A F}^{\mathrm{T}} \boldsymbol{K}_{1} \boldsymbol{X}_{A F}-\boldsymbol{X}_{2 F}^{\mathrm{T}} \boldsymbol{K}_{2} \boldsymbol{X}_{2 F} \\
= & -W_{F} \\
\leq & 0
\end{aligned}
$$

and according to Equation (19), $W_{F}=0$ if and only if $\boldsymbol{X}_{1 F}=\mathbf{0}_{4 n \times 1}, \boldsymbol{X}_{2 F}=\mathbf{0}_{3 n \times 1}$.

Theorem 1. Considering the spacecraft attitude tracking synchronization system governed by (26) and (28), if graph $\mathcal{G}(t)$ satisfies assumption 1 , by designing the control torque composed by (24) and (31), the backstepping state $\boldsymbol{X}_{1 \mathrm{~F}}, \boldsymbol{X}_{2 \mathrm{~F}}$ can globally asymptotically converge to $\boldsymbol{X}_{1 \mathrm{~F}}=\mathbf{0}_{4 n \times 1}$, $X_{2 \mathrm{~F}}=\mathbf{0}_{3 n \times 1}$, i.e., the Object 2 is achieved. 
Proof of Theorem 1. Theorem 1 can be obtained from (32)

Remark 2. Although $T$ is treated as a whole in the derivation, in the actual calculation, all the FS $i, i=2 \ldots n$ calculate their controller $\boldsymbol{T}_{i} \in \boldsymbol{R}^{3}$ independently. It can be obtained according to Equation (31) and the fact that $\boldsymbol{K}_{2}$ and $\boldsymbol{J}$ are block diagonal matrices and $\boldsymbol{X}_{A F}, \boldsymbol{X}_{2 F}, \boldsymbol{\omega}$ and $\dot{f}_{n}\left(X_{A F}\right)$ are column stack matrices. For each FS $i, i=2 \ldots n$, the external information required for constructing its controller $\boldsymbol{T}_{i}$ is only normalized information $\boldsymbol{\zeta}_{i}^{(1)}$ and $\boldsymbol{s}_{i}^{(2)}$. Thus, as Section 3.2 illustrated, the broadcasting states from every neighbor $j,(j, i) \in \varepsilon$ at consecutive broadcasting instants $t_{k} \in \boldsymbol{R}_{\geq 0}, k \in \boldsymbol{N}_{\geq 0}$ should be only $\boldsymbol{Q}_{j}(k), \boldsymbol{X}_{1 F, j}(k)$, and 1 .

Remark 3. The control error of $\boldsymbol{X}_{1 F}$ and $\boldsymbol{X}_{2 F}$ can be decreased and the convergence rate can be increased by increasing $K_{1}$ and $\boldsymbol{K}_{2}$ properly in a certain range.

\subsubsection{Discrete Control Scheme}

In space missions, the continuous model of communication is unrealistic. The spacecraft update information at every communicating instant $t_{k} \in R_{\geq 0}, k \in N_{\geq 0}$. Therefore, at instant $t_{k}$, the control torque can be modified according to the information transmitted by their neighbors as Equation (15), such that $\boldsymbol{X}_{1 \mathrm{~F}}(k), \boldsymbol{P}_{2}(k)$ and $\boldsymbol{X}_{2 \mathrm{~F}}(k)$ of the spacecraft are updated by $\boldsymbol{Q}(k)$ and $\boldsymbol{\omega}(k)$ according to Equations (16)-(18) and (24) as

$$
\begin{aligned}
& \boldsymbol{X}_{1 \mathrm{~F}, i}(k)=\boldsymbol{Q}_{i}(k)-\boldsymbol{s}_{i}^{(1)}(k), i=1 \ldots n, \\
& \boldsymbol{X}_{1 \mathrm{~F}}(k)=\left[\boldsymbol{X}_{1 \mathrm{~F}, 1}^{\mathrm{T}}(k) \ldots \boldsymbol{X}_{1 \mathrm{~F}, n}^{\mathrm{T}}(k)\right]^{\mathrm{T}} \in \boldsymbol{R}^{4 n \times 1} \text {, } \\
& \boldsymbol{P}_{2}(k)=\operatorname{diag}\left(\Xi\left[\boldsymbol{Q}_{1}\right](k) \ldots \Xi\left[\boldsymbol{Q}_{n}\right](k)\right) \text {, } \\
& \boldsymbol{X}_{\mathrm{AF}}(k)=\boldsymbol{P}_{2}(k)\left(\boldsymbol{L}_{A}(k) \otimes \mathbf{I}_{4}\right) \operatorname{diag}\left(\mathbf{I}_{4} \boldsymbol{\zeta}_{1}^{\prime}(k), \ldots, \mathbf{I}_{4} \boldsymbol{\zeta}_{n}^{\prime}(k)\right)^{-1} \boldsymbol{X}_{1 \mathrm{~F}}(k) \\
& =\boldsymbol{P}_{2}(k)\left[\begin{array}{c}
\frac{\sum_{(j, 1) \in \varepsilon}[\mathcal{C}(k)]_{j, 1}\left(\boldsymbol{X}_{1 \mathrm{~F}, 1}(k)-\boldsymbol{X}_{1 \mathrm{~F}, j}(k)\right)}{\sum_{(j, 1) \in \varepsilon}[\mathcal{C}(k)]_{j, 1}} \\
\vdots \\
\frac{\sum_{(j, n) \in \varepsilon}[\mathcal{C}(k)]_{j, n}\left(\boldsymbol{X}_{1 \mathrm{~F}, n}(k)-\boldsymbol{X}_{1 \mathrm{~F}, j}(k)\right)}{\sum_{(j, n) \in \varepsilon}[\mathcal{C}(k)]_{j, n}}
\end{array}\right] \\
& =\boldsymbol{P}_{2}(k)\left[\begin{array}{c}
\boldsymbol{X}_{1 \mathrm{~F}, 1}(k)-\boldsymbol{\varsigma}_{1}^{(2)}(k) \\
\vdots \\
\boldsymbol{X}_{1 \mathrm{~F}, n}(k)-\boldsymbol{\varsigma}_{n}^{(2)}(k)
\end{array}\right] \text {, } \\
& \boldsymbol{X}_{2 \mathrm{~F}}(k)=\boldsymbol{\omega}(k)-f_{n}\left(\boldsymbol{X}_{\mathrm{AF}}(k)\right) \in \boldsymbol{R}^{3 n \times 1} \text {, } \\
& \boldsymbol{X}_{2 \mathrm{~F}, i}(k)=\left[\left[\boldsymbol{X}_{2 \mathrm{~F}}(k)\right]_{3 i-2,1},\left[\left[\boldsymbol{X}_{2 \mathrm{~F}}(k)\right]_{3 i-1,1},\left[\left[\boldsymbol{X}_{2 \mathrm{~F}}(k)\right]_{3 i, 1}\right]^{\mathrm{T}}, i=1 \ldots n\right. \text {. }\right.
\end{aligned}
$$

Correspondingly, during all periods $\left[t_{k}, t_{k+1}\right)$, considering Equations (24), (31) and Equation (33), the stabilizing function and input torque for the FS are

$$
\begin{aligned}
f_{n}\left(\boldsymbol{X}_{\mathrm{AF}}(k)\right) & =-\boldsymbol{K}_{1} \boldsymbol{X}_{\mathrm{AF}}(k), \\
\boldsymbol{T}(k) & =-\boldsymbol{X}_{\mathrm{AF}}(k)-\boldsymbol{K}_{2} \boldsymbol{X}_{2 F}(k) \\
& +\boldsymbol{\omega}(k) \times \boldsymbol{J} \boldsymbol{\omega}(k)+\boldsymbol{J} \frac{f_{n}\left(\boldsymbol{X}_{\mathrm{AF}}(k+1)\right)-f_{n}\left(\boldsymbol{X}_{\mathrm{AF}}(k)\right)}{\Delta t}, \\
\boldsymbol{T}_{i} & =\left[\begin{array}{c}
{[\boldsymbol{T}]_{3 i-2,1}} \\
{[\boldsymbol{T}]_{3 i-1,1}} \\
{[\boldsymbol{T}]_{3 i, 1}}
\end{array}\right], i=1 \ldots n
\end{aligned}
$$

where $\boldsymbol{X}_{\mathrm{AF}}(k+1)$ is estimated by $\boldsymbol{X}_{\mathrm{AF}}(k)+\Delta t \dot{\boldsymbol{X}}_{\mathrm{AF}}(k), \boldsymbol{T}_{1} \in \boldsymbol{R}^{3}$ is redundant for the FS to achieve the attitude synchronization with the LS. Therefore, $\boldsymbol{T}_{i} \in \boldsymbol{R}^{3}, i=2 \ldots n$ is the input torque for each FS $i$. 
By using Euler's first-order approximation [40], the kinematics (26) and (28) during all periods $\left[t_{k}, t_{k+1}\right)$ can be rewritten as:

$$
\begin{aligned}
\frac{\boldsymbol{X}_{1 \mathrm{~F}}(k+1)-\boldsymbol{X}_{1 \mathrm{~F}}(k)}{\Delta t} & =\frac{1}{2} \mathcal{L}^{\mathrm{T}}\left(t_{k}\right) \boldsymbol{P}_{2}^{\mathrm{T}}(k)\left(f_{n}\left(\boldsymbol{X}_{\mathrm{AF}}(k)\right)+\boldsymbol{X}_{2 \mathrm{~F}}(k)\right) \\
\boldsymbol{J} \frac{\boldsymbol{X}_{2 \mathrm{~F}}(k+1)-\boldsymbol{X}_{2 \mathrm{~F}}(k)}{\Delta t} & =\boldsymbol{T}(k)-\boldsymbol{\omega}(k) \times \boldsymbol{J} \boldsymbol{\omega}(k)-\boldsymbol{J} \frac{f_{n}\left(\boldsymbol{X}_{\mathrm{AF}}(k+1)\right)-f_{n}\left(\boldsymbol{X}_{\mathrm{AF}}(k)\right)}{\Delta t}
\end{aligned}
$$

Theorem 2. Considering the spacecraft modeled by (35), if graph $\mathcal{G}\left(t_{k}\right)$ satisfy Assumption 1, by designing the control torque as Equation (34) with the following four constraints

1. $\lambda_{\min }\left(\Delta t \boldsymbol{K}_{1}-\Delta t^{2} \boldsymbol{J}^{-2}\right)>\lambda_{\max }\left(\frac{\Delta t^{2} \mathcal{D}^{2}}{4} \boldsymbol{K}_{1} \boldsymbol{K}_{1}\right)$,

2. $\quad \lambda_{\min }\left(2 \Delta t \boldsymbol{J}^{-1} \boldsymbol{K}_{2}-\Delta t^{2} \boldsymbol{K}_{2} \boldsymbol{J}^{-2} \boldsymbol{K}_{2}\right)>\frac{\Delta t^{2} \mathcal{D}^{2}}{4}$

3. $\mathcal{Z}_{\mathrm{F}} \mathcal{Z}_{\mathrm{F}}^{\mathrm{T}} \geq 0$, where $\mathcal{Z}_{\mathrm{F}}=\frac{\Delta t \boldsymbol{K}_{1} \boldsymbol{P}_{2}(k) \mathcal{L}(k) \mathcal{L}^{\mathrm{T}}(k) \boldsymbol{P}_{2}^{\mathrm{T}}(k)}{4}+\boldsymbol{J}^{-1}-\Delta t \boldsymbol{J}^{-2} \boldsymbol{K}_{2}-\frac{1}{2}$,

4. $-\Delta t^{2} \mathcal{D}^{2}+\lambda_{\min }\left(2 \Delta t \boldsymbol{J}^{-1} \boldsymbol{K}_{2}-\Delta t^{2} \boldsymbol{K}_{2} \boldsymbol{J}^{-2} \boldsymbol{K}_{2}\right)>\Delta t \frac{\left(\lambda_{\max }\left(\boldsymbol{J}^{-1}-\Delta t \boldsymbol{J}^{-2} \boldsymbol{K}_{2}-\frac{1}{2}\right)+\lambda_{\max }\left(\Delta t \boldsymbol{K}_{1}\right) \mathcal{D}^{2}\right)^{2}}{\lambda_{\min }\left(\boldsymbol{K}_{1}-\Delta t \boldsymbol{J}^{-2}\right)+\left(-\lambda_{\max }\left(\Delta t \boldsymbol{K}_{1}\right) \mathcal{D}^{2} \lambda_{\max }\left(\boldsymbol{K}_{1}\right)\right)^{2}}$, the backstepping state $\boldsymbol{X}_{1 \mathrm{~F}}, \boldsymbol{X}_{2 \mathrm{~F}}$ can $(\beta, A)$-practically stably converge to $\boldsymbol{X}_{1 \mathrm{~F}}=\mathbf{0}_{4 n \times 1}$, $X_{2 \mathrm{~F}}=\mathbf{0}_{3 n \times 1}$, i.e., the Object 2 is achieved.

Proof of Theorem 2. Equations (34) and (35) can be rewritten as

$$
X_{\mathrm{F}}(k+1)=A_{\mathrm{F}}(k) X_{\mathrm{F}}(k),
$$

where

$$
\begin{aligned}
\boldsymbol{X}_{\mathrm{F}} & =\left[\begin{array}{c}
\boldsymbol{X}_{1 \mathrm{~F}} \\
\boldsymbol{X}_{2 \mathrm{~F}}
\end{array}\right] \in \boldsymbol{R}^{7 n}, \\
\boldsymbol{A}_{\mathrm{F}}(k) & =\left[\begin{array}{cc}
\mathbf{I}_{4 n}-\frac{\Delta t \mathcal{L}^{\mathrm{T}}(k) \boldsymbol{P}_{2}^{\mathrm{T}}(k) \boldsymbol{K}_{1} \boldsymbol{P}_{2}(k) \mathcal{L}(k)}{2} & \frac{\Delta t \mathcal{L}^{\mathrm{T}}(k) \boldsymbol{P}_{2}^{\mathrm{T}}(k)}{2} \\
-\Delta t \boldsymbol{J}^{-1} \boldsymbol{P}_{2}(k) \mathcal{L}(k) & \mathbf{I}_{3 n}-\Delta t \boldsymbol{J}^{-1} \boldsymbol{K}_{2}
\end{array}\right] \in \boldsymbol{R}^{7 n \times 7 n,}
\end{aligned}
$$

define the Lyapunov function $V_{\mathrm{F}}=\boldsymbol{X}_{\mathrm{F}}^{\mathrm{T}} \boldsymbol{X}_{\mathrm{F}}$, then $\forall \boldsymbol{X}_{\mathrm{F}} \neq \mathbf{0}_{7 n \times 1}$, the following equation holds

$$
V_{\mathrm{F}}(k+1)-V_{\mathrm{F}}(k)=\boldsymbol{X}_{\mathrm{F}}^{\mathrm{T}}(k)\left(A_{\mathrm{F}}^{\mathrm{T}}(k) \boldsymbol{A}_{\mathrm{F}}(k)-\mathbf{I}_{7 n}\right) \boldsymbol{X}_{\mathrm{F}}(k)
$$

then according to Lemma 7 , if $\mathbf{I}_{7 n}-A_{\mathrm{F}}^{\mathrm{T}}(k) \boldsymbol{A}_{\mathrm{F}}(k)$ is positive definite $\forall \boldsymbol{X}_{\mathrm{F}} \neq \mathbf{0}_{7 n \times 1}$, by choosing $K_{1}, K_{2}$ and $\Delta t$, the Euler's first-order approximation (36) is EGAS. Therefore, considering Lemma 4, the accurate discrete approximation of the spacecraft attitude tracking synchronization system governed by (26) and (28) is $(\beta, A)$-practically stable, i.e., $\boldsymbol{X}_{\mathrm{F}}$ can converge to the neighborhood of the origin $(\beta, A)$-practically stably.

$$
\text { Represent }\left[\mathbf{I}_{7 n}-\boldsymbol{A}_{\mathrm{F}}^{\mathrm{T}}(k) \boldsymbol{A}_{\mathrm{F}}(k)\right]_{1,1^{\prime}}\left[\mathbf{I}_{7 n}-\boldsymbol{A}_{\mathrm{F}}^{\mathrm{T}}(k) \boldsymbol{A}_{\mathrm{F}}(k)\right]_{1,2^{\prime}}\left[\mathbf{I}_{7 n}-\boldsymbol{A}_{\mathrm{F}}^{\mathrm{T}}(k) \boldsymbol{A}_{\mathrm{F}}(k)\right]_{2,1^{\prime}}
$$
$\left[\mathbf{I}_{7 n}-A_{\mathrm{F}}^{\mathrm{T}}(k) \boldsymbol{A}_{\mathrm{F}}(k)\right]_{2,2}$ as $\mathcal{P}_{\mathrm{F}} \in \boldsymbol{R}^{4 n \times 4 n}, \mathcal{Q}_{\mathrm{F}} \in \boldsymbol{R}^{4 n \times 3 n}, \mathcal{Q}_{\mathrm{F}}^{\mathrm{T}} \in \boldsymbol{R}^{3 n \times 4 n}$, and $\mathcal{R}_{\mathrm{F}} \in \boldsymbol{R}^{3 n \times 3 n}$, respectively, then $\mathbf{I}_{7 n}-\boldsymbol{A}_{\mathrm{F}}^{\mathrm{T}}(k) \boldsymbol{A}_{\mathrm{F}}(k)$ is denoted as

$$
\mathbf{I}_{7 n}-\mathbf{A}_{\mathrm{F}}^{\mathrm{T}}(k) \mathbf{A}_{\mathrm{F}}(k)=\left[\begin{array}{cc}
\mathcal{P}_{\mathrm{F}} & \mathcal{Q}_{\mathrm{F}} \\
\mathcal{Q}_{\mathrm{F}}^{\mathrm{T}} & \mathcal{R}_{\mathrm{F}}
\end{array}\right]
$$

according to Lemma $7, \forall \boldsymbol{X}_{\mathrm{F}} \neq \mathbf{0}_{7 n \times 1}, \mathcal{P}_{\mathrm{F}}, \mathcal{R}_{\mathrm{F}}$ and $\mathcal{R}_{\mathrm{F}}-\mathcal{Q}_{\mathrm{F}}^{\mathrm{T}} \mathcal{P}_{\mathrm{F}}^{-1} \mathcal{Q}_{\mathrm{F}}$ should be positive definite by choosing $K_{1}, K_{2}$ and $\Delta t$.

i. $\quad \mathcal{P}_{\mathrm{F}}=\Delta t \boldsymbol{M}_{\mathrm{F}}(k)-\frac{\Delta t^{2} \boldsymbol{M}_{\mathrm{F}}(k)^{\mathrm{T}} \boldsymbol{M}_{\mathrm{F}}(k)}{4}-\Delta t^{2} \mathcal{L}^{\mathrm{T}}(k) \boldsymbol{P}_{2}^{\mathrm{T}}(k) \boldsymbol{J}^{-2} \boldsymbol{P}_{2}(k) \mathcal{L}(k)$, where $\boldsymbol{M}_{\mathrm{F}}=\mathcal{L}^{\mathrm{T}} \boldsymbol{P}_{2}^{\mathrm{T}} \boldsymbol{K}_{1} \boldsymbol{P}_{2} \mathcal{L}$. 
As $\forall \mathcal{X}_{\mathrm{F}} \in \boldsymbol{R}^{4 n}$

$$
\begin{aligned}
& \mathcal{X}_{\mathrm{F}}^{\mathrm{T}}\left(\Delta t \boldsymbol{M}_{\mathrm{F}}(k)-\Delta t^{2} \mathcal{L}^{\mathrm{T}}(k) \boldsymbol{P}_{2}^{\mathrm{T}}(k) \boldsymbol{J}^{-2} \boldsymbol{P}_{2}(k) \mathcal{L}(k)\right) \mathcal{X}_{\mathrm{F}} \\
& =\Delta t \mathcal{X}_{\mathrm{F}}^{\mathrm{T}} \mathcal{L}^{\mathrm{T}}(k) \boldsymbol{P}_{2}^{\mathrm{T}}(k)\left(\boldsymbol{K}_{1}-\Delta t \boldsymbol{J}^{-2}\right) \boldsymbol{P}_{2}(k) \mathcal{L}(k) \mathcal{X}_{\mathrm{F}} \\
& \geq \lambda_{\min }\left(\Delta t \boldsymbol{K}_{1}-\Delta t^{2} \boldsymbol{J}^{-2}\right) \boldsymbol{Y}^{\mathrm{T}} \boldsymbol{Y}
\end{aligned}
$$

and

$$
\begin{aligned}
& \mathcal{X}_{\mathrm{F}}^{\mathrm{T}} \frac{\Delta t^{2} \boldsymbol{M}_{\mathrm{F}}(k)^{\mathrm{T}} \boldsymbol{M}_{\mathrm{F}}(k)}{4} \mathcal{X}_{\mathrm{F}} \\
& =\Delta t^{2} \mathcal{X}_{\mathrm{F}}^{\mathrm{T}} \mathcal{L}^{\mathrm{T}}(k) \boldsymbol{P}_{2}^{\mathrm{T}}(k) \frac{\boldsymbol{K}_{1} \boldsymbol{P}_{2}(k) \mathcal{L}(k) \mathcal{L}^{\mathrm{T}}(k) \boldsymbol{P}_{2}^{\mathrm{T}}(k) \boldsymbol{K}_{1}}{4} \boldsymbol{P}_{2}(k) \mathcal{L}(k) \mathcal{X}_{\mathrm{F}} \\
& \leq \lambda_{\max }\left(\frac{\Delta t^{2} \mathcal{L}(k) \mathcal{L}^{\mathrm{T}}(k)}{4}\right) \boldsymbol{Y}^{\mathrm{T}} \boldsymbol{K}_{1} \boldsymbol{K}_{1} \boldsymbol{Y} \\
& \leq \lambda_{\max }\left(\frac{\Delta t^{2} \mathcal{L}(k) \mathcal{L}^{\mathrm{T}}(k)}{4}\right) \lambda_{\max }\left(\boldsymbol{K}_{1} \boldsymbol{K}_{1}\right) \boldsymbol{Y}^{\mathrm{T}} \boldsymbol{Y},
\end{aligned}
$$

where $\boldsymbol{Y}=\boldsymbol{P}_{2}(k) \mathcal{L}(k) \mathcal{X}_{\mathrm{F}}$, and the fact (20) is used here. According to [41], we get the following inequality

$$
\lambda_{\max }(\mathcal{L}(k)) \leq \max (d(i)+d(j) \mid i, j \in \mathcal{N},(i, j) \in \varepsilon),
$$

represent $\max (d(i)+d(j) \mid i, j \in \mathcal{N},(i, j) \in \varepsilon)$ with $\mathcal{D}$, Equation (40) can be rewritten as

$$
\mathcal{X}_{\mathrm{F}}^{\mathrm{T}} \frac{\Delta t^{2} \boldsymbol{M}_{\mathrm{F}}(k)^{\mathrm{T}} \boldsymbol{M}_{\mathrm{F}}(k)}{4} \mathcal{X}_{\mathrm{F}} \leq \lambda_{\max }\left(\frac{\Delta t^{2} \mathcal{D}^{2}}{4} \boldsymbol{K}_{1} \boldsymbol{K}_{1}\right) \boldsymbol{Y}^{\mathrm{T}} \boldsymbol{Y} .
$$

According to Equations (39) and (41), by choosing

$$
\lambda_{\min }\left(\Delta t \boldsymbol{K}_{1}-\Delta t^{2} \boldsymbol{J}^{-2}\right)>\lambda_{\max }\left(\frac{\Delta t^{2} \mathcal{D}^{2}}{4} \boldsymbol{K}_{1} \boldsymbol{K}_{1}\right),
$$

$\mathcal{P}_{\mathrm{F}}$ is ensured to be positive definite $\forall \boldsymbol{X}_{\mathrm{F}} \neq \mathbf{0}_{7 n \times 1}$.

ii. $\quad \mathcal{R}_{\mathrm{F}}=\frac{-\Delta t^{2} \boldsymbol{P}_{2}(k) \mathcal{L}(k) \mathcal{L}^{\mathrm{T}}(k) \boldsymbol{P}_{2}^{\mathrm{T}}(k)}{4}+2 \Delta t \boldsymbol{J}^{-1} \boldsymbol{K}_{2}-\Delta t^{2} \boldsymbol{K}_{2} \boldsymbol{J}^{-2} \boldsymbol{K}_{2}$ should be positive definite $\forall \boldsymbol{X}_{\mathrm{F}} \neq \mathbf{0}_{7 n \times 1}$. Which means, by choosing $\boldsymbol{K}_{2}$ and $\Delta t$ to satisfy

$$
\lambda_{\min }\left(2 \Delta t \boldsymbol{J}^{-1} \boldsymbol{K}_{2}-\Delta t^{2} \boldsymbol{K}_{2} \boldsymbol{J}^{-2} \boldsymbol{K}_{2}\right)>\frac{\Delta t^{2} \mathcal{D}^{2}}{4}
$$

$\mathcal{R}_{\mathrm{F}}$ is ensured to be positive definite $\forall \boldsymbol{X}_{\mathrm{F}} \neq \mathbf{0}_{7 n \times 1}$.

iii. Define

$$
G_{\mathrm{F}}=\mathcal{R}_{\mathrm{F}}-\mathcal{Q}_{\mathrm{F}}^{\mathrm{T}} \mathcal{P}_{\mathrm{F}}^{-1} \mathcal{Q}_{\mathrm{F}}
$$

according to Lemma 7,

$$
\lambda_{\text {min }}\left(\mathcal{R}_{\mathrm{F}}\right)>\lambda_{\text {max }}\left(\mathcal{Q}_{\mathrm{F}}^{\mathrm{T}} \mathcal{P}_{\mathrm{F}}^{-1} \mathcal{Q}_{\mathrm{F}}\right),
$$

should be guaranteed. With some calculations

$$
\mathcal{Q}_{\mathrm{F}}^{\mathrm{T}} \mathcal{P}_{\mathrm{F}}^{-1} \mathcal{Q}_{\mathrm{F}}=\Delta t \mathcal{Z}_{\mathrm{F}}^{\mathrm{T}} \mathcal{Y}_{\mathrm{F}}^{-1} \mathcal{Z}_{\mathrm{F}},
$$

where

$$
\begin{aligned}
& \mathcal{Z}_{\mathrm{F}}=\frac{\Delta t \boldsymbol{K}_{1} \boldsymbol{P}_{2}(k) \mathcal{L}(k) \mathcal{L}^{\mathrm{T}}(k) \boldsymbol{P}_{2}^{\mathrm{T}}(k)}{4}+\boldsymbol{J}^{-1}-\Delta t \boldsymbol{J}^{-2} \boldsymbol{K}_{2}-\frac{1}{2}, \\
& \mathcal{Y}_{\mathrm{F}}=\boldsymbol{K}_{1}-\frac{\Delta t \boldsymbol{K}_{1} \boldsymbol{P}_{2}(k) \mathcal{L}(k) \mathcal{L}^{\mathrm{T}}(k) \boldsymbol{P}_{2}^{\mathrm{T}}(k) \boldsymbol{K}_{1}}{4}-\Delta t \boldsymbol{J}^{-2} .
\end{aligned}
$$

Hence, Equation (44) can be rewritten as

$$
\lambda_{\text {min }}\left(\mathcal{R}_{\mathrm{F}}\right)>\lambda_{\text {max }}\left(\Delta t \mathcal{Z}_{\mathrm{F}}^{\mathrm{T}} \mathcal{Y}_{\mathrm{F}}^{-1} \mathcal{Z}_{\mathrm{F}}\right)
$$


As

$$
\begin{aligned}
\Delta t \lambda\left(\mathcal{Z}_{\mathrm{F}}^{\mathrm{T}} \mathcal{Y}_{\mathrm{F}}^{-1} \mathcal{Z}_{\mathrm{F}}\right) & =\Delta t \lambda\left(\left(\mathcal{Z}_{\mathrm{F}}^{\mathrm{T}}\right)^{-1} \mathcal{Z}_{\mathrm{F}}^{\mathrm{T}} \mathcal{Y}_{\mathrm{F}}^{-1} \mathcal{Z}_{\mathrm{F}} \mathcal{Z}_{\mathrm{F}}^{\mathrm{T}}\right) \\
& =\Delta t \lambda\left(\mathcal{Y}_{\mathrm{F}}^{-1} \mathcal{Z}_{\mathrm{F}} \mathcal{Z}_{\mathrm{F}}^{\mathrm{T}}\right)
\end{aligned}
$$

Equation (47) turns into

$$
\lambda_{\min }\left(\mathcal{R}_{\mathrm{F}}\right)>\lambda_{\max }\left(\Delta t \mathcal{Y}_{\mathrm{F}}^{-1} \mathcal{Z}_{\mathrm{F}} \mathcal{Z}_{\mathrm{F}}^{\mathrm{T}}\right)
$$

According to Lemma 9, if

$$
\mathcal{Z}_{\mathrm{F}} \mathcal{Z}_{\mathrm{F}}^{\mathrm{T}} \geq \mathbf{0}_{3 n \times 3 n}
$$

is ensured by choosing $K_{1}$ and $K_{2}$, then the upper bound of $\lambda_{\max }\left(\Delta t \mathcal{Y}_{\mathrm{F}}^{-1} \mathcal{Z}_{\mathrm{F}} \mathcal{Z}_{\mathrm{F}}^{\mathrm{T}}\right)$ can be bounded as

$$
\lambda_{\max }\left(\Delta t \mathcal{Y}_{\mathrm{F}}^{-1} \mathcal{Z}_{\mathrm{F}} \mathcal{Z}_{\mathrm{F}}^{\mathrm{T}}\right) \leq \lambda_{\max }\left(\Delta t \mathcal{Y}_{\mathrm{F}}^{-1}\right) \lambda_{\max }\left(\mathcal{Z}_{\mathrm{F}} \mathcal{Z}_{\mathrm{F}}^{\mathrm{T}}\right)
$$

According to Lemma 8, we obtain the following inequalities

$$
\begin{gathered}
\lambda_{\min }\left(\mathcal{R}_{\mathrm{F}}\right) \geq \\
\geq \lambda_{\min }\left(-\frac{\Delta t^{2} \boldsymbol{P}_{2}(k) \mathcal{L}(k) \mathcal{L}^{\mathrm{T}}(k) \boldsymbol{P}_{2}^{\mathrm{T}}(k)}{4}\right) \\
+\lambda_{\min }\left(2 \Delta t \boldsymbol{J}^{-1} \boldsymbol{K}_{2}-\Delta t^{2} \boldsymbol{K}_{2} \boldsymbol{J}^{-2} \boldsymbol{K}_{2}\right) \\
\lambda_{\max }\left(\Delta t \mathcal{Y}_{\mathrm{F}}^{-1}\right) \leq \Delta t\left(\lambda_{\min }\left(\boldsymbol{K}_{1}-\Delta t \boldsymbol{J}^{-2}\right)+\lambda_{\min }\left(-\frac{\Delta t \boldsymbol{K}_{1} \boldsymbol{P}_{2}(k) \mathcal{L}(k) \mathcal{L}^{\mathrm{T}}(k) \boldsymbol{P}_{2}^{\mathrm{T}}(k) \boldsymbol{K}_{1}}{4}\right)\right)^{-1} \\
\lambda_{\max }\left(\mathcal{Z}_{\mathrm{F}}\right) \leq \quad \lambda_{\max }\left(\boldsymbol{J}^{-1}-\Delta t \boldsymbol{J}^{-2} \boldsymbol{K}_{2}-\frac{1}{2}\right)+\lambda_{\max }\left(\frac{\Delta t \boldsymbol{K}_{1} \boldsymbol{P}_{2}(k) \mathcal{L}(k) \mathcal{L}^{\mathrm{T}}(k) \boldsymbol{P}_{2}^{\mathrm{T}}(k)}{4}\right)
\end{gathered}
$$

According to Lemma 9,

$$
\begin{gathered}
\lambda_{\max }\left(\frac{\Delta t \boldsymbol{K}_{1} \boldsymbol{P}_{2}(k) \mathcal{L}(k) \mathcal{L}^{\mathrm{T}}(k) \boldsymbol{P}_{2}^{\mathrm{T}}(k)}{4}\right) \leq \lambda_{\max }\left(\Delta t \boldsymbol{K}_{1}\right) \lambda_{\max }\left(\frac{\boldsymbol{P}_{2}(k) \mathcal{L}(k) \mathcal{L}^{\mathrm{T}}(k) \boldsymbol{P}_{2}^{\mathrm{T}}(k)}{4}\right) \\
\lambda_{\max }\left(\frac{\Delta t \boldsymbol{K}_{1} \boldsymbol{P}_{2}(k) \mathcal{L}(k) \mathcal{L}^{\mathrm{T}}(k) \boldsymbol{P}_{2}^{\mathrm{T}}(k) \boldsymbol{K}_{1}}{4}\right) \leq \lambda_{\max }\left(\Delta t \boldsymbol{K}_{1}\right) \lambda_{\max }\left(\frac{\boldsymbol{P}_{2}(k) \mathcal{L}(k) \mathcal{L}^{\mathrm{T}}(k) \boldsymbol{P}_{2}^{\mathrm{T}}(k)}{4}\right) \lambda_{\max }\left(\boldsymbol{K}_{1}\right) .
\end{gathered}
$$

According to Lemma 10,

$$
\lambda_{\max }\left(\frac{\boldsymbol{P}_{2}(k) \mathcal{L}(k) \mathcal{L}^{\mathrm{T}}(k) \boldsymbol{P}_{2}^{\mathrm{T}}(k)}{4}\right) \leq \mathcal{D}^{2}
$$

Then Equation (52) can be rewritten as

$$
\begin{aligned}
\lambda_{\min }\left(\mathcal{R}_{\mathrm{F}}\right) & \geq-\Delta t^{2} \mathcal{D}^{2}+\lambda_{\min }\left(2 \Delta t \boldsymbol{J}^{-1} \boldsymbol{K}_{2}-\Delta t^{2} \boldsymbol{K}_{2} \boldsymbol{J}^{-2} \boldsymbol{K}_{2}\right) \\
\lambda_{\max }\left(\Delta t \mathcal{Y}_{\mathrm{F}}^{-1}\right) & \leq \Delta t\left(\lambda_{\min }\left(\boldsymbol{K}_{1}-\Delta t \boldsymbol{J}^{-2}\right)+\left(-\lambda_{\max }\left(\Delta t \boldsymbol{K}_{1}\right) \mathcal{D}^{2} \lambda_{\max }\left(\boldsymbol{K}_{1}\right)\right)\right)^{-1} \\
\lambda_{\max }\left(\mathcal{Z}_{\mathrm{F}}\right) & \leq \lambda_{\max }\left(\boldsymbol{J}^{-1}-\Delta t \boldsymbol{J}^{-2} \boldsymbol{K}_{2}-\frac{1}{2}\right)+\lambda_{\max }\left(\Delta t \boldsymbol{K}_{1}\right) \mathcal{D}^{2}
\end{aligned}
$$

By combining Equations (51) and (53), we get the following inequality

$$
\begin{aligned}
& \lambda_{\max }\left(\Delta t \mathcal{Y}_{\mathrm{F}}^{-1} \mathcal{Z}_{\mathrm{F}} \mathcal{Z}_{\mathrm{F}}^{\mathrm{T}}\right) \\
\leq & \lambda_{\max }\left(\Delta t \mathcal{Y}_{\mathrm{F}}^{-1}\right) \lambda_{\max }\left(\mathcal{Z}_{\mathrm{F}} \mathcal{Z}_{\mathrm{F}}^{\mathrm{T}}\right) \\
\leq & \lambda_{\max }\left(\Delta t \mathcal{Y}_{\mathrm{F}}^{-1}\right)\left(\lambda_{\max }\left(\mathcal{Z}_{\mathrm{F}}\right)\right)^{2} \\
\leq & \Delta t \frac{\left(\lambda_{\max }\left(\boldsymbol{J}^{-1}-\Delta t \boldsymbol{J}^{-2} \boldsymbol{K}_{2}-\frac{1}{2}\right)+\lambda_{\max }\left(\Delta t \boldsymbol{K}_{1}\right) \mathcal{D}^{2}\right)^{2}}{\lambda_{\min }\left(\boldsymbol{K}_{1}-\Delta t \boldsymbol{J}^{-2}\right)+\left(-\lambda_{\max }\left(\Delta t \boldsymbol{K}_{1}\right) \mathcal{D}^{2} \lambda_{\max }\left(\boldsymbol{K}_{1}\right)\right)^{\prime}}
\end{aligned}
$$


hence Equations (44), (47), and (49) can be ensured by choosing

$$
\begin{gathered}
-\Delta t^{2} \mathcal{D}^{2}+\lambda_{\min }\left(2 \Delta t \boldsymbol{J}^{-1} \boldsymbol{K}_{2}-\Delta t^{2} \boldsymbol{K}_{2} \boldsymbol{J}^{-2} \boldsymbol{K}_{2}\right) \\
>\quad \Delta t \frac{\left(\lambda_{\max }\left(\boldsymbol{J}^{-1}-\Delta t \boldsymbol{J}^{-2} \boldsymbol{K}_{2}-\frac{1}{2}\right)+\lambda_{\max }\left(\Delta t \boldsymbol{K}_{1}\right) \mathcal{D}^{2}\right)^{2}}{\lambda_{\min }\left(\boldsymbol{K}_{1}-\Delta t \boldsymbol{J}^{-2}\right)+\left(-\lambda_{\max }\left(\Delta t \boldsymbol{K}_{1}\right) \mathcal{D}^{2} \lambda_{\max }\left(\boldsymbol{K}_{1}\right)\right)},
\end{gathered}
$$

In conclusion, if Equations (42), (43), (50), and (55) are satisfied, one can reach the requirement $\mathrm{I}_{7 n}-A_{\mathrm{F}}^{\mathrm{T}}(k) A_{\mathrm{F}}(k)$ is positive definite $\forall X_{\mathrm{F}} \neq \mathbf{0}_{7 n \times 1}$. Then according to Lemma 3, the Euler's first-order approximation (36) is EGAS. Therefore, considering Lemma 4, the accurate discrete approximation of the spacecraft attitude tracking synchronization system governed by (26) and (28) is $(\beta, \boldsymbol{A})$-practically stable, i.e., $\boldsymbol{X}_{\mathrm{F}}$ can converge to the neighborhood of the origin $(\beta, A)$-practically stably. Physically, the attitude of the spacecraft in the swarm can track the space moving target synchronously.

\section{Simulation Results}

In order to adapt to the limits of different propulsion systems, two simulation cases that satisfy Assumption 1 are simulated in this section. The orbit elements of the target and spacecraft are listed in Table 1, and spacecraft 1 acts as the LS. The attitude quaternion $Q_{1}$ of the LS is calculated according to the relative position of the target and LS using the methodology in Section 3.1, and the initial attitude $Q_{i}(0)$ of spacecraft $i=2 \ldots 6$ is given randomly. Then the FS will maneuver to achieve the attitude synchronization with the LS. As Section 2.5 illustrated, the aforementioned attitude tracking synchronization in this paper can be converted into a typical spacecraft attitude cooperative tracking problem, namely, the control algorithm in this paper can be applied to a wide range of attitude cooperative tracking problems.

Table 1. Orbit elements of the target and spacecraft.

\begin{tabular}{ccccccc}
\hline Orbit Elements & Target & Leader 1 & Follower 2 & Follower 3 & Follower 4 & Follower 5 \\
\hline Semi-major axis $a(\mathrm{~km})$ & 6790 & 6900 & 6900 & 6900 & 6900 & $1 \times 10^{-9}$ \\
\hline Eccentricity $e$ & 0.0169 & $1 \times 10^{-9}$ & $1 \times 10^{-9}$ & $1 \times 10^{-9}$ & 3000 & 30 \\
\hline Inclination $i\left(^{\circ}\right)$ & 96 & 30 & 30 & 150 & 150 & 150 \\
\hline $\begin{array}{c}\text { Right Ascension of the } \\
\text { Ascending Node } \Omega\left({ }^{\circ}\right)\end{array}$ & 45 & 150 & 30 & 30 & 30 & 30 \\
\hline Argument of Perigee $\omega\left(^{\circ}\right)$ & 30 & 30 & 7.01 & 7.02 & 6.99 \\
\hline Initial true anomaly $\Theta_{0}\left(^{\circ}\right)$ & 75 & 7 & & 30 \\
\hline
\end{tabular}

The communication graph is fixed as Figure 3 and channel fading coefficient $[\mathcal{C}(t)]_{i, j}$ are generated independently and identically distributed in $(0,1]$ by MATLAB.

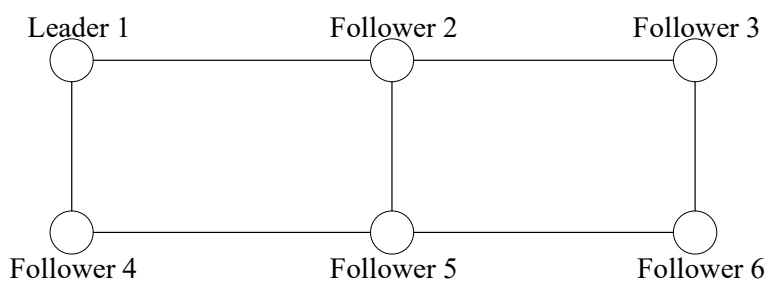

Figure 3. Graph $\mathcal{G}$ for six spacecraft in the swarm.

The momentum of inertia of all the spacecraft are set as

$$
J_{i}=\left[\begin{array}{ccc}
8 & 0.02 & 0.01 \\
0.02 & 8.1 & 0.01 \\
0.01 & 0.01 & 8.2
\end{array}\right] \mathrm{kg} \cdot \mathrm{m}^{2}, i=1 \ldots 6
$$


The interval $\Delta t$ between update instants is set as $0.1 \mathrm{~s}$, and each spacecraft receives three signals as Equation (14) at instants $t_{k} \in \boldsymbol{R}_{\geq 0}, k \in \boldsymbol{N}_{\geq 0}$. Traditionally, a decimal requires 8 bytes, namely, 64 bits. Therefore, using the methodology in this paper, the channel required by each spacecraft for attitude control is $640 \mathrm{~b} / \mathrm{s}$, which can be satisfied by the inter-spacecraft communication capability more than enough [42]. In contrast, when using the traditional OCAM, every spacecraft needs to receive three signals from each neighbor respectively. In other words, if a spacecraft has $n$ neighbors, the required bit rate is $n$ times the size of that in this paper.

\subsection{The Control Case Utilizing Liquid Propulsion (LP) Systems}

The thrust that LP systems can provide is usually within $1 \mathrm{~N}$ [43]. In order to meet the requirement, the parameters $K_{1}$ and $K_{2}$ in control inputs are chosen as $K_{1}=0.9 \mathbf{I}_{18}$, and $K_{2}=3 \mathbf{I}_{18}$. Figure 4 shows the attitude of each spacecraft in the swarm using Euler angle $\boldsymbol{\alpha}_{i}=\left[\Phi_{i}, \theta_{i}, \Psi_{i}\right]^{\mathrm{T}} \in \boldsymbol{R}^{3}, i=1 \ldots 6$. Figure 5 shows the attitude error of each spacecraft in the swarm using Euler angle $\boldsymbol{\alpha}_{\mathrm{e}, i}=\left[\Phi_{e, i}, \theta_{e, i}, \Psi_{e, i}\right]^{\mathrm{T}} \in \boldsymbol{R}^{3}, i=1 \ldots 6$. The relationships between Euler angle and quaternion can be found in [29]. Clearly, the attitude tracking synchronization errors of each spacecraft will decrease within $\left[-0.1^{\circ}, 0.1^{\circ}\right]$, by using the control law given in Sections 3.1 and 3.3. As can be seen in Figure 6, the proposed control torques $\boldsymbol{T}_{i}=\left[T_{c \Phi i}, T_{c \theta i}, T_{c \Psi i}\right]^{\mathrm{T}} \in \boldsymbol{R}^{3}, i=1 \ldots 6$ are within $[-1 \mathrm{~N} \cdot \mathrm{m}, 1 \mathrm{~N} \cdot \mathrm{m}]$, and is practicable by combining the LP systems [43] and the actuator in [44]. Hence, the communication and control scheme proposed in this paper are verified.

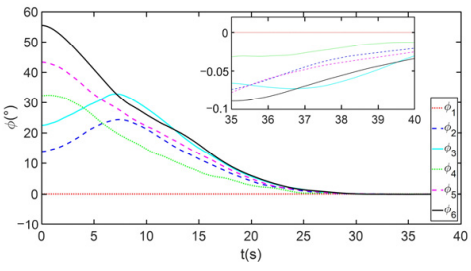

(a)

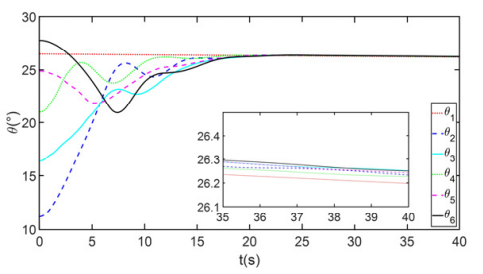

(b)

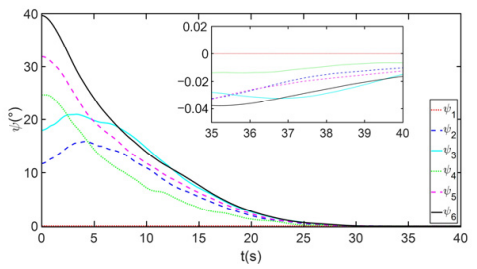

(c)

Figure 4. The attitude of the swarm utilizing LP systems: (a) Roll attitude; (b) Pitch attitude; (c) Yaw attitude.

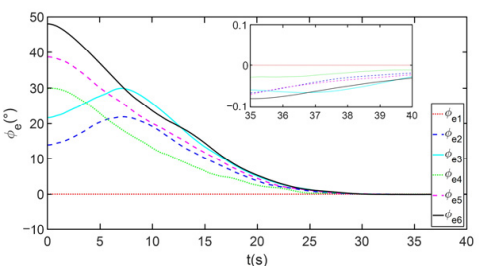

(a)

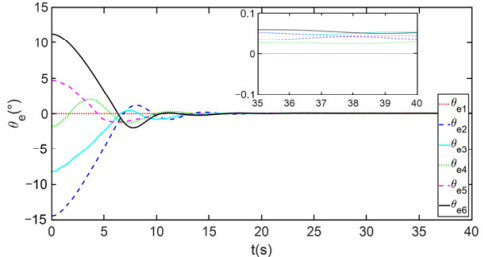

(b)

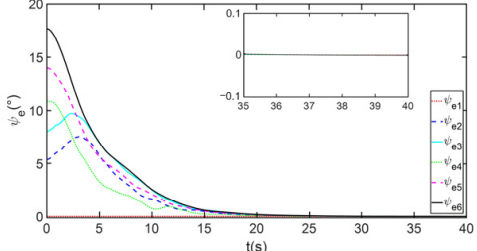

(c)

Figure 5. The attitude tracking synchronization error of the swarm utilizing LP systems: (a) Roll error; (b) Pitch error; (c) Yaw error.

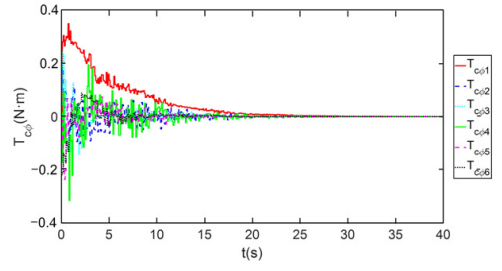

(a)

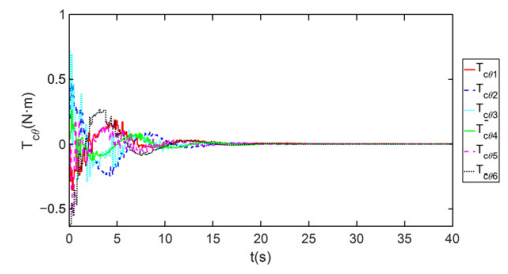

(b)

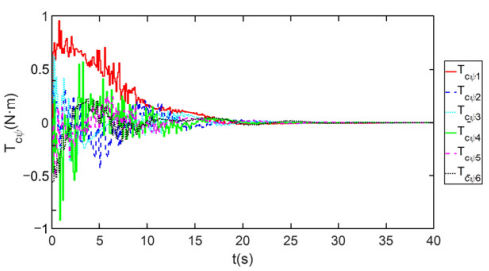

(c)

Figure 6. The control torque for the swarm utilizing LP systems: (a) Roll torque; (b) Pitch torque; (c) Yaw torque. 


\subsection{The Control Case Utilizing Solid Rocket Propulsion (SRP) Systems or Nuclear Propulsion (NP) Systems}

The SRP systems usually provide the thrust within $76 \mathrm{~N}$, and have flown on the SPINSAT mission [43]. The nuclear propulsion (NP) systems can provide the thrust up to $1.2 \times 10^{7} \mathrm{~N}$, which remains the best option for interplanetary journeys towards far-off planets [45-47]. Accordingly, the parameters $K_{1}$ and $K_{2}$ in control inputs are chosen as $\boldsymbol{K}_{1}=4.2 \mathbf{I}_{18}$, and $\boldsymbol{K}_{2}=18 \mathbf{I}_{18}$. Figure 7 shows the attitude of each spacecraft in the swarm using Euler angle $\boldsymbol{\alpha}_{i}=\left[\Phi_{i}, \theta_{i}, \Psi_{i}\right]^{\mathrm{T}} \in \boldsymbol{R}^{3}, i=1 \ldots 6$. Figure 8 shows the attitude error of each spacecraft in the swarm using Euler angle $\boldsymbol{\alpha}_{\mathrm{e}, i}=\left[\Phi_{e, i}, \theta_{e, i}, \Psi_{e, i}\right]^{\mathrm{T}} \in \boldsymbol{R}^{3}, i=1 \ldots 6$. Clearly, the attitude tracking synchronization errors of each spacecraft will decrease within $\left[-0.1^{\circ}, 0.1^{\circ}\right]$, by using the control law given in Sections 3.1 and 3.3. As can be seen in Figure 9, the proposed control torques $\boldsymbol{T}_{i}=\left[T_{c \Phi i}, T_{c \theta i}, T_{c \Psi i}\right]^{\mathrm{T}} \in \boldsymbol{R}^{3}, i=1 \ldots 6$ are with $[-20 \mathrm{~N} \cdot \mathrm{m}, 20 \mathrm{~N} \cdot \mathrm{m}]$, and are practicable by combining the SRP system or NP system and the actuator in [43]. Hence, the communication and control scheme proposed in this paper are verified.

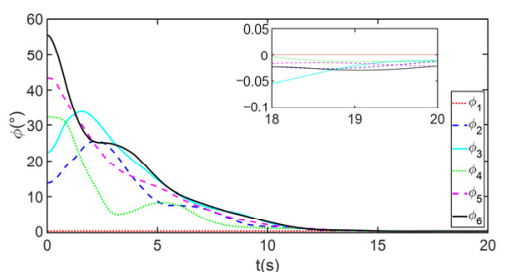

(a)

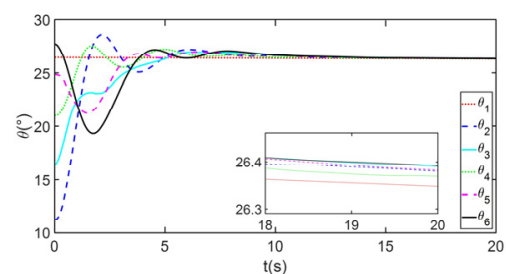

(b)

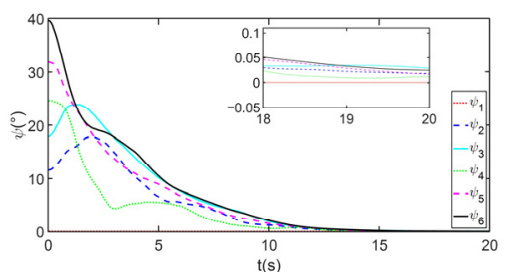

(c)

Figure 7. The attitude of the swarm utilizing SRP systems or NP systems: (a) Roll attitude; (b) Pitch attitude; (c) Yaw attitude.

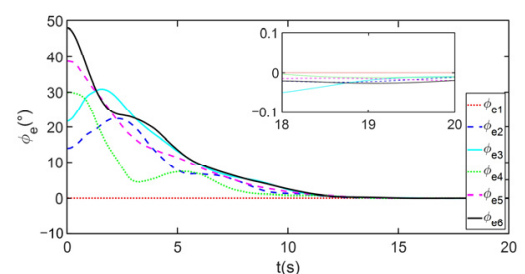

(a)

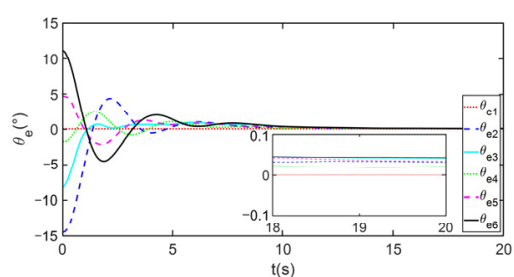

(b)

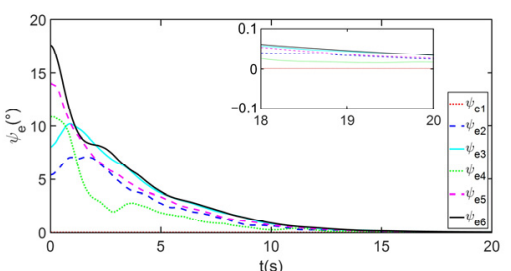

(c)

Figure 8. The attitude tracking synchronization error of the swarm utilizing SRP systems or NP systems: (a) Roll error; (b) Pitch error; (c) Yaw error.

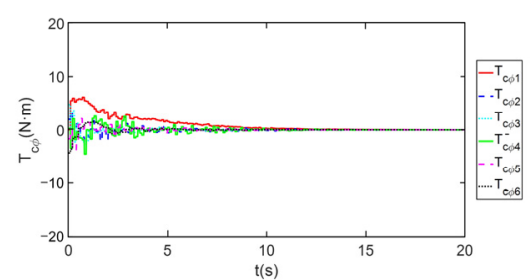

(a)

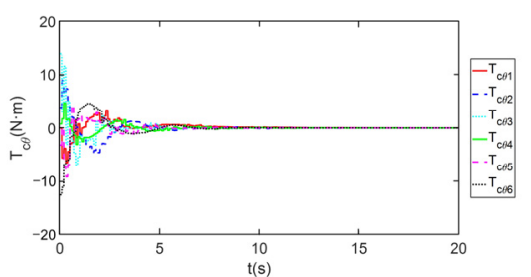

(b)

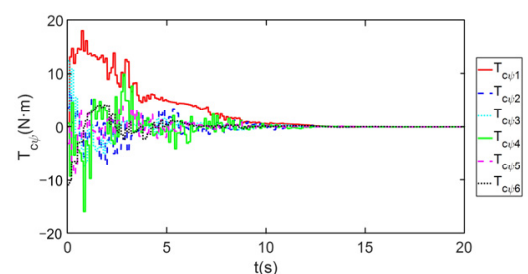

(c)

Figure 9. The control torque for the swarm utilizing SRP systems or NP systems: (a) Roll torque; (b) Pitch torque; (c) Yaw torque.

\section{Conclusions}

Discrete spacecraft swarm attitude tracking synchronization exploiting interference for a moving target is researched in this paper. Information interference is used for information transmitting to solve the challenge of limited communication in a spacecraft 
swarm. Compared with time-multiplexing OCAM or frequency-multiplexing OCAM, the algorithm can achieve high speed and save communication resources proportionally to the number of spacecraft in practice. The control law based on the backstepping control technique in this paper also deals with the unknown channel attenuation problem when exploiting information interference. Simulation shows using the control method proposed in this paper, the attitude error is within $0.1^{\circ}$, while the control torque level can be adjusted by selecting control parameters $K_{1}$ and $K_{2}$ to adapt to different propulsion systems. Additionally, the channel required by each spacecraft for attitude control is $640 \mathrm{~b} / \mathrm{s}$, namely, the methodology proposed in this paper will perform in engineering. In addition, the method in this paper is applicable to typical spacecraft attitude cooperative tracking problem.

Author Contributions: Methodology, P.L. and M.Z.; software, H.L.; validation, P.L. and X.W.; formal analysis, P.L.; investigation, D.L.; resources, X.W. and Y.L.; data curation, M.Z.; writing-original draft preparation, P.L. and X.W.; writing—review and editing, M.Z.; visualization, D.L.; supervision, Y.L.; project administration, H.L.; funding acquisition, P.L. and D.L. All authors have read and agreed to the published version of the manuscript.

Funding: This work has been supported by the Chinese Government Scholarship (No.201906830037) and Interdisciplinary Innovation Foundation for Graduates, NUAA (No.KXKCXJJ202010).

Institutional Review Board Statement: Not applicable.

Informed Consent Statement: Not applicable.

Data Availability Statement: Not applicable.

Conflicts of Interest: The authors declare no conflict of interest.

\section{References}

1. Nosseir, A.E.S.; Cervone, A.; Pasini, A. Modular impulsive green monopropellant propulsion system (mimps-g): For cubesats in leo and to the moon. Aerospace 2021, 8, 169. [CrossRef]

2. Kramer, A.; Bangert, P.; Schilling, K. Uwe-4: First electric propulsion on a $1 \mathrm{u}$ cubesat in-orbit experiments and characterization. Aerospace 2020, 7, 98. [CrossRef]

3. Bandyopadhyay, S.; Foust, R.; Subramanian, G.P.; Chung, S.-J.; Hadaegh, F.Y. Review of formation flying and constellation missions using nanosatellites. J. Spacecr. Rocket. 2016, 53, 567-578. [CrossRef]

4. Utschick, W. Communications in Interference Limited Networks; Springer: Cham, Switzerland, 2016.

5. Truszkowski, W.; Hinchey, M.; Rash, J.; Rouff, C. NASA's swarm missions: The challenge of building autonomous software. IT Prof. 2004, 6, 47-52. [CrossRef]

6. Liu, H.; Xie, G.; Wang, L. Necessary and sufficient conditions for containment control of networked multi-agent systems. Automatica 2012, 48, 1415-1422. [CrossRef]

7. Borzone, T.; Morărescu, I.-C.; Jungers, M.; Boc, M.; Janneteau, C. Hybrid framework for consensus in fleets of non-holonomic robots. In Proceedings of the 2018 Annual American Control Conference (ACC), Milwaukee, WI, USA, 27-29 June 2018; pp. 4299-4304.

8. Chen, Z.; Chen, X. Robust sampled-data output synchronization of nonlinear heterogeneous multi-agents. IEEE Trans. Autom. Control 2016, 62, 1458-1464. [CrossRef]

9. Abdessameud, A.; Tayebi, A.; Polushin, I.G. Leader-follower synchronization of euler-lagrange systems with time-varying leader trajectory and constrained discrete-time communication. IEEE Trans. Autom. Control 2016, 62, 2539-2545. [CrossRef]

10. Furieri, L.; Kamgarpour, M. The value of communication in designing robust distributed controllers. arXiv 2017, arXiv:1711.05324.

11. Olfati-Saber, R.; Fax, J.; Murray, R. Consensus and cooperation in networked multi-agent systems. Proc. IEEE 2007, 95, 215-233. [CrossRef]

12. Mei, J.; Ren, W.; Chen, J.; Ma, G. Distributed adaptive coordination for multiple lagrangian systems under a directed graph without using neighbors velocity information. Automatica 2013, 49, 1723-1731. [CrossRef]

13. Zhao, S.; Zelazo, D. Bearing rigidity and almost global bearing-only formation stabilization. IEEE Trans. Autom. Control 2015, 61, 1255-1268. [CrossRef]

14. Ren, W.; Beard, R.W.; Atkins, E.M. Information consensus in multivehicle cooperative control. IEEE Control Syst. Mag. 2007, 27, 71-82.

15. Jie, M.; Wei, R.; Jie, C.; Anderson, B.D.O. Consensus of linear multi-agent systems with fully distributed control gains under a general directed graph. In Proceedings of the 53rd IEEE Conference on Decision and Control, Los Angeles, CA, USA, 15-17 December 2014; pp. 2993-2998. 
16. Movric, K.H.; Lewis, F.L. Cooperative optimal control for multi-agent systems on directed graph topologies. IEEE Trans. Autom. Control 2014, 59, 769-774. [CrossRef]

17. Lewis, F.L.; Cui, B.; Ma, T.; Song, Y.; Zhao, C. Heterogeneous multi-agent systems: Reduced-order synchronization and geometry. IEEE Trans. Autom. Control 2016, 61, 1391-1396. [CrossRef]

18. Ren, W. Distributed leaderless consensus algorithms for networked euler-lagrange systems. Int. J. Control 2009, 82, 2137-2149. [CrossRef]

19. Chen, L.-M.; Li, C.-J.; Mei, J.; Ma, G.-F. Adaptive cooperative formation-containment control for networked euler-lagrange systems without using relative velocity information. IET Control Theory Appl. 2017, 11, 1450-1458. [CrossRef]

20. Du, H.; Li, S. Attitude synchronization for flexible spacecraft with communication delays. IEEE Trans. Autom. Control 2016, 61, 3625-3630. [CrossRef]

21. Mehrabian, A.; Khorasani, K. Distributed and cooperative quaternion-based attitude synchronization and tracking control for a network of heterogeneous spacecraft formation flying mission. J. Frankl. Inst. 2015, 352, 3885-3913. [CrossRef]

22. Zhu, Z.; Guo, Y. Adaptive coordinated attitude control for spacecraft formation with saturating actuators and unknown inertia. J. Frankl. Inst. 2019, 356, 1021-1037. [CrossRef]

23. Yu, Y.; Liu, W.; Yang, Z.; Miao, C.; Jiang, W. A distributed consensus protocol for attitude synchronization and tracking of multiple spacecraft on directed graphs. In Proceedings of the 2019 IEEE 15th International Conference on Control and Automation (ICCA), Edinburgh, UK, 16-19 July 2019; pp. 881-886.

24. Molinari, F.; Stańczak, S.; Raisch, J. Exploiting the superposition property of wireless communication for max-consensus problems in multi-agent systems. IFAC-PapersOnLine 2018, 51, 176-181. [CrossRef]

25. Molinari, F.; Stanczak, S.; Raisch, J. Exploiting the superposition property of wireless communication for average consensus problems in multi-agent systems. In Proceedings of the 2018 European Control Conference (ECC), Limassol, Cyprus, 12-15 June 2018; pp. 1766-1772.

26. Cao, Y.; Yu, W.; Ren, W.; Chen, G. An overview of recent progress in the study of distributed multi-agent coordination. IEEE Trans. Ind. Inform. 2013, 9, 427-438. [CrossRef]

27. Junkins, J.L.; Schaub, H. Analytical Mechanics of Space Systems; American Institute of Aeronautics and Astronautics: Reston, VA, USA, 2009.

28. Ickes, B.P. A new method for performing digital control system attitude computations using quaternions. AIAA J. 1970, 8, 13-17. [CrossRef]

29. Sidi, M.J. Spacecraft Dynamics and Control: A Practical Engineering Approach; Cambridge University Press: Cambridge, UK, 1997; Volume 7.

30. Xing, Y.; Cao, X.; Zhang, S.; Guo, H.; Wang, F. Relative position and attitude estimation for satellite formation with coupled translational and rotational dynamics. Acta Astronaut. 2010, 67, 455-467. [CrossRef]

31. Molinari, F.; Raisch, J. Exploiting wireless interference for distributively solving linear equations. IFAC-PapersOnLine 2020, 53, 2999-3006. [CrossRef]

32. Goldenbaum, M.; Boche, H.; Stańczak, S. Harnessing interference for analog function computation in wireless sensor networks IEEE Trans. Signal Process. 2013, 61, 4893-4906. [CrossRef]

33. Nešić, D.; Teel, A.R.; Kokotović, P.V. Sufficient conditions for stabilization of sampled-data nonlinear systems via discrete-time approximations. Syst. Control Lett. 1999, 38, 259-270. [CrossRef]

34. Arcak, M.; Nešić, D. A framework for nonlinear sampled-data observer design via approximate discrete-time models and emulation. Automatica 2004, 40, 1931-1938. [CrossRef]

35. Gentle, J.E. Matrix Algebra: Theory, Computations, and Applications in Statistics; Springer Science \& Business Media: Berlin/Heidelberg, Germany, 2007.

36. De Oliveira, M.C. Fundamentals of Linear Control, 1st ed.; Cambridge University Press: Cambridge, UK, 2017. Available online: http:/ /gen.lib.rus.ec/book/index.php?md5=8e37a4532fa49eedf0cde87167a81903 (accessed on 7 June 2020).

37. Marshall, A.W.; Olkin, I. Inequalities: Theory of Majorization and Its Applications, 1st ed.; Mathematics in Science and Engineering 143; Academic Press: Cambridge, MA, USA, 1979. Available online: http://gen.lib.rus.ec/book/index.php?md5=0b2ac25bbd5a4 6c14c037295582b2438 (accessed on 7 June 2020).

38. Zhang, F.; Zhang, Q. Eigenvalue inequalities for matrix product. IEEE Trans. Autom. Control 2006, 51, 1506-1509. [CrossRef]

39. Molinari, F.; Raisch, J. Efficient consensus-based formation control with discrete-time broadcast updates. arXiv 2019, arXiv:1903.07906.

40. Iserles, A. A First Course in the Numerical Analysis of Differential Equations; Cambridge Texts in Applied Mathematics; Cambridge University Press: Cambridge, UK, 1996. Available online: http://gen.lib.rus.ec/book/index.php?md5=b9acbfe1d21dfcf1aa32e9 95624f76b1 (accessed on 7 June 2020).

41. Wen-Ren, H.K. A sharp upper bound on the largest eigenvalue of the laplacian matrix of a graph. Linear Algebra Appl. 2002, 347, 123-129.

42. Farrag, A.; Othman, S.; Mahmoud, T.; El Raffiei, A.Y. Satellite swarm survey and new conceptual design for Earth observation applications. Egypt. J. Remote Sens. Space Sci. 2021, 24, 47-54. [CrossRef]

43. Tummala, A.R.; Dutta, A. An overview of cube-satellite propulsion technologies and trends. Aerospace 2017, 4, 58. [CrossRef] 
44. Wu, Y.H.; Han, F.; Zheng, M.H.; Wang, F.; Hua, B.; Chen, Z.M.; Cheng, Y.H. Attitude tracking control for a space moving target with high dynamic performance using hybrid actuator. Aerosp. Sci. Technol. 2018, 78, 102-117. [CrossRef]

45. Frisbee, R.H. Limits of interstellar flight technology. Front. Propuls. Sci. 2009, 227, 31-126.

46. Gibson, M.A.; Mason, L.S.; Bowman, C.L.; Poston, D.I.; McClure, P.R.; Creasy, J.; Robinson, C. Development of NASA's Small Fission Power System for Science and Human Exploration. In Proceedings of the Joint Propulsion Conference, $\mathrm{Cleveland,} \mathrm{OH}$ USA, 28 July 2014. (GRC-E-DAA-TN17266)

47. Mazouffre, S. Electric propulsion for satellites and spacecraft: Established technologies and novel approaches. Plasma Sources Sci. Technol. 2016, 25, 033002. [CrossRef] 